\title{
Projected BCS theory for the unification of antiferromagnetism and strongly correlated superconductivity
}

\author{
Hyunwoong Kwon ${ }^{1}$ and Kwon Park (1) 1,2,3,* \\ ${ }^{1}$ Quantum Universe Center, Korea Institute for Advanced Study, Seoul 02455, Korea \\ ${ }^{2}$ School of Physics, Korea Institute for Advanced Study, Seoul 02455, Korea \\ ${ }^{3}$ Department of Physics, Harvard University, Cambridge, Massachusetts 02138, USA
}

(Received 4 August 2020; revised 19 January 2022; accepted 25 January 2022; published 11 February 2022)

\begin{abstract}
A close connection between antiferromagnetism and superconductivity is at the core of high-temperature superconductivity. Here, we put forward the projected BCS theory for the unification of antiferromagnetism and strongly correlated superconductivity. Specifically, it is shown that, with the $d$-wave pairing symmetry, the projected BCS theory can provide excellent trial states at general doping for the exact ground states of the $t-J$ model in the square lattice, generating a unified theory of high-temperature superconductivity as a continuous function of hole concentration. A key to the success of the projected BCS theory is an accurate treatment of the strong correlation between Cooper pairs, which not only causes the breakdown of superconductivity itself at half filling but also defines the precise nature of strongly correlated superconductivity at moderate doping. Also, via the proper incorporation of particle number fluctuations, the projected BCS theory allows direct computation of the superconducting order parameter, shedding important light on the pseudogap phenomenon.
\end{abstract}

DOI: 10.1103/PhysRevResearch.4.013116

\section{INTRODUCTION}

Despite the large variety in material properties, there is a certain list of common features robust across various hightemperature superconductors. One of the most salient features in such a list is the proximity between antiferromagnetism and superconductivity. An important question is exactly how these two phenomena are connected together.

The spin singlet is the smallest unit of antiferromagnetism. Simply put, the BCS state is the antisymmetrized condensate of spin-singlet electron pairs, i.e., Cooper pairs. Therefore it is natural to expect that antiferromagnetism, at least with the short-range order, is closely connected with superconductivity in some form. The resonating valence bond (RVB) state is the trial state constructed under this rationale. Specifically, the RVB state is the projected BCS state with all components containing doubly occupied sites projected out [1-8]. Known as the Gutzwiller projection, this projection implements the effect of infinitely strong on-site repulsive interaction, while it can be also partially imposed [9-11].

A problem is that, at half filling, the RVB state reduces to a spin liquid rather than the true antiferromagnet with the long-range order, i.e., the Néel state. In principle, quantum antiferromagnets can host various different types of spin liquids and valence bond solids in two dimensions [12]. Experimen-

\footnotetext{
*kpark@kias.re.kr

Published by the American Physical Society under the terms of the Creative Commons Attribution 4.0 International license. Further distribution of this work must maintain attribution to the author(s) and the published article's title, journal citation, and DOI.
}

tally, however, all known high-temperature superconductors tend to have the Néel order at half filling. If so, it would mean that superconductivity is fundamentally incompatible with the long-range antiferromagnetic order. Is this true?

Here, we show that it is possible to construct a unified theory of antiferromagnetism (AF) and strongly correlated superconductivity (SCSC). A breakthrough of the unified theory is that the Gutzwiller projection is directly applied onto the BCS Hamiltonian itself, not just its ground state. Specifically, the BCS Hamiltonian is directly diagonalized in the projected Hilbert space under the no-double-occupancy constraint $[13,14]$. Let us call this theory the projected BCS theory.

To provide the validity of the projected BCS theory, we perform exact diagonalization of the projected BCS Hamiltonian and compare the so-obtained exact ground states with those of the $t-J$ model. Actually, a similar exact-diagonalization technique has been previously used to analyze the projected BCS Hamiltonian [13,14]. While generating promising results, however, this previous analysis suffered from various problems due to critical finite-size effects. Here, we overcome these problems by devising the proper overlap valid in the presence of particle number fluctuations.

As a result, it is shown that the projected BCS theory provides excellent trial states for the exact ground states of the $t-J$ model in the square lattice for a wide range of hole concentration. In particular, it is proved analytically that the exact ground states of the $t-J$ model and the projected BCS theory are entirely equivalent at half filling. At moderate doping, the projected BCS theory with the $d$-wave pairing symmetry continues to provide excellent trial states for the $t-J$ model, demonstrating the merit of unifying AF and SCSC, as emphasized by the $\mathrm{SO}(5)$ theory [15]. 
Also, properly incorporating particle number fluctuations, the projected BCS theory allows direct computation of the superconducting order parameter, providing concrete evidence for the split between the "bare" and real superconducting order parameters at low doping, which can in principle explain one of the great mysteries in high-temperature superconductivity, the pseudogap phenomenon.

Finally, the projected BCS theory can be also used to investigate the relevance of the $s$-wave pairing symmetry as well as the geometrical frustration to high-temperature superconductivity.

\section{RESULTS}

\section{A. High-temperature superconductivity and the fractional quantum Hall effect}

Arguably, there are two main pillars in the research of strongly correlated electron systems, high-temperature superconductivity and the fractional quantum Hall effect (FQHE). Our work is motivated by a remarkable similarity between the RVB state [1] for high-temperature superconductivity and the composite fermion (CF) state [16] for the FQHE.

To begin with, the RVB state is the electron-electron paired state projected onto the Hilbert space with no double occupancy:

$$
\psi_{\mathrm{RVB}}=\mathcal{P}_{\mathrm{G}} \psi_{\mathrm{BCS}},
$$

where $\mathcal{P}_{\mathrm{G}}$ is the Gutzwiller projection operator, and $\psi_{\mathrm{BCS}}$ is the BCS wave function. In view of the Gutzwiller projection, it is convenient to write $\psi_{\mathrm{BCS}}$ in the real space:

$$
\psi_{\mathrm{BCS}}=\mathcal{A}\left[\phi\left(\mathbf{r}_{1}-\mathbf{r}_{2}\right) \phi\left(\mathbf{r}_{3}-\mathbf{r}_{4}\right) \cdots \phi\left(\mathbf{r}_{N-1}-\mathbf{r}_{N}\right)\right],
$$

where $\mathcal{A}$ is the antisymmetrization operators and $\phi\left(\mathbf{r}_{i}-\mathbf{r}_{j}\right)$ is the pair function between the $i$ th and $j$ th electrons. Specifically, $\phi\left(\mathbf{r}_{i}-\mathbf{r}_{j}\right)$ can be written as follows:

$$
\phi\left(\mathbf{r}_{i}-\mathbf{r}_{j}\right)=\varphi\left(\mathbf{r}_{i}-\mathbf{r}_{j}\right) \frac{1}{\sqrt{2}}(|\uparrow \downarrow\rangle-|\downarrow \uparrow\rangle)_{i j},
$$

where $\varphi\left(\mathbf{r}_{i}-\mathbf{r}_{j}\right)$ is the real-space part of the pair function.

The next step is to perform $\mathcal{P}_{\mathrm{G}}$ by removing the doubly occupied components from $\psi_{\mathrm{BCS}}$. Concretely, after the Gutzwiller projection, the amplitude of $\psi_{\mathrm{RVB}}$ can be written for a given spin distribution $\left\{\mathbf{r}_{\uparrow}, \mathbf{r}_{\downarrow}\right\}$ as follows:

$$
\psi_{\mathrm{RVB}}\left(\left\{\mathbf{r}_{\uparrow}, \mathbf{r}_{\downarrow}\right\}\right)=\operatorname{det}\left[\varphi\left(\mathbf{r}_{i}-\mathbf{r}_{j}\right)\right],
$$

where $i$ and $j$ run though the coordinates of all spin up and down electrons in $\left\{\mathbf{r}_{\uparrow}, \mathbf{r}_{\downarrow}\right\}$, respectively [3]. See Appendix A for details.

It is important to note that, expressed in terms of the determinant, the above form of $\psi_{\mathrm{RVB}}$ tells us that Cooper pairs can be treated as being more or less weakly interacting, while the pairing correlation becomes somewhat weakened. The precise nature of the weakened pairing correlation depends on the hole concentration.

Similarly, the CF state is the electron-vortex bound state projected onto the lowest Landau level (LL):

$$
\psi_{\mathrm{CF}}=\mathcal{P}_{\mathrm{LLL}} \psi_{\mathrm{J}},
$$

where $\mathcal{P}_{\text {LLL }}$ is the lowest LL projection operator, and $\psi_{\mathrm{J}}$ is the Jastrow-factor correlated wave function,

$$
\psi_{\mathrm{J}}=\prod_{i<j} J_{i j}^{2} \operatorname{det}\left[\phi_{n m}\left(z_{i}^{*}, z_{i}\right)\right],
$$

where $J_{i j}=z_{i}-z_{j}$ is the Jastrow factor with $z=x+i y$, and $\phi_{n m}\left(z_{i}^{*}, z_{i}\right)$ is the wave function for the LL eigenstate under a reduced effective magnetic field with $n$ and $m$ being the effective LL index and the angular momentum quantum number, respectively. Note that, here, we focus on the circular gauge in the planar geometry.

The next step is to perform $\mathcal{P}_{\text {LLL }}$ by appropriately splitting the Jastrow factors and absorbing the split parts into the determinant. Concretely, after the lowest LL projection, the $\mathrm{CF}$ state can be written as follows:

$$
\psi_{\mathrm{CF}}=\operatorname{det}\left[\widehat{\phi}_{n m}\left(\frac{\partial}{\partial z_{i}}, z_{i}\right) \prod_{j \neq i} J_{i j}\right],
$$

where $\widehat{\phi}_{n m}$ is the normal-ordered operator derived from $\phi_{n m}$ with $z^{*}$ replaced by $\partial / \partial z$ :

$$
\widehat{\phi}_{n m}\left(\frac{\partial}{\partial z_{i}}, z_{i}\right)=: \phi_{n m}\left(z_{i}^{*} \rightarrow \frac{\partial}{\partial z_{i}}, z_{i}\right):
$$

where the normal ordering means that differential operators go all the way to the front of the expression. See Ref. [17] for details.

It is important to note that the above form of $\psi_{\mathrm{CF}}$ is also given in terms of the determinant, meaning that CFs can be also treated as being more or less weakly interacting. Similar to the Gutzwiller projection, however, the electron-vortex binding becomes weakened by the lowest LL projection. In other words, vortices are somewhat detached from electrons after the lowest LL projection, while the precise degree of detachment depends on the filling factor.

In conclusion, the great merit of both RVB and CF states lies in the fact that relevant quasiparticles, Cooper pairs and CFs, respectively, can be treated as being more or less weakly interacting. Specifically, the "plain vanilla" version of the RVB theory assuming basically noninteracting Cooper pairs can provide a semiquantitative understanding of hightemperature superconductivity [8]. Similarly, the FQHE can be understood as the integer quantum Hall effect of weakly interacting CFs [16], explaining various essential properties of the FQHE [17].

As mentioned above, however, both Gutzwiller and lowest LL projections weaken paired and bound states, respectively, by generating the residual interaction between quasiparticles. A natural question is what happens if the residual interaction gets too strong to cause the breakdown of otherwise weakly interacting quasiparticles.

Actually, the RVB state is bound to break down in the vicinity of half filling, where the phase fluctuation of Cooper pairs becomes too strong, and thus the long-range antiferromagetic order takes over. Meanwhile, the $\mathrm{CF}$ state breaks down when promoted to the second LL, where the residual interaction between CFs becomes attractive [18-22], and consequently the even-denominator FQHE state can occur at filling factor $v=5 / 2$ [23]. What would be the wave function 
capturing such strongly correlated states and how can we obtain them?

Fundamentally, we need to improve the weakly interacting part of the wave function. For example, $\psi_{\mathrm{BCS}}$ and $\psi_{\mathrm{J}}$ can be systematically improved to some extents via the second quantization formalism. Concretely, various gauge theories have been constructed for high-temperature superconductivity by implementing $\mathcal{P}_{\mathrm{G}}$ in the slave particle formalism [24]. Similarly, the Chern-Simons gauge theory has been constructed for the FQHE by implementing $\mathcal{P}_{\text {LLL }}$ in both mean-field and one-loop levels [25-27]. Despite some reasonable successes, however, these approaches are not properly equipped to capture the sheer breakdown of weakly interacting quasiparticles, which requires a much more accurate treatment of strong correlation. To this end, we get inspirations from the aforementioned even-denominator FQHE at filling factor $v=5 / 2$.

It is generally believed that the $5 / 2 \mathrm{FQHE}$ state is a paired quantum Hall state induced by the emergent, attractive correlation between CFs [18-22]. Among the most promising trial states for the 5/2 FQHE state are the Moore-Read (MR) Pfaffian state $[18,19]$, the particle-hole $(\mathrm{PH})$ conjugate of the MR Pfaffian state (also known as the anti-Pfaffian state), and their PH-symmetrized combination [28-30]. While the MR Pfaffian state can be written explicitly in the planar geometry, it is generally much more convenient to analyze the model Hamiltonian, which generates the MR Pfaffian state as the exact ground state.

Such a model Hamiltonian can be obtained as the threebody repulsive interaction, $H_{3}$, imposing an energy penalty to the closest-packed clusters of three electrons. Ultimately, checking the validity of the MR Pfaffian state amounts to computing the overlap between the exact ground states of the Coulomb interaction and $\mathrm{H}_{3}$ [28-30]. In summary, the weakly interacting $\mathrm{CF}$ state can be improved upon by considering the exact ground state of the model Hamiltonian $\mathrm{H}_{3}$.

Inspired by this use of $H_{3}$ generating the MR Pfaffian state, here, we consider the projected BCS Hamiltonian, which can play a role of the model Hamiltonian generating the improved trial state beyond the RVB state.

\section{B. Projected BCS Hamiltonian}

The projected BCS Hamiltonian can be written as follows:

$$
H_{\mathrm{PBCS}}=\mathcal{P}_{\mathrm{G}}\left(H_{t}+H_{\Delta}+H_{\mu}\right) \mathcal{P}_{\mathrm{G}},
$$

where

$$
\begin{aligned}
H_{t} & =-t \sum_{\langle i, j\rangle, \sigma}\left(c_{i \sigma}^{\dagger} c_{j \sigma}+\text { H.c. }\right) \\
H_{\Delta} & =\sum_{\langle i, j\rangle} \Delta_{i j}\left(c_{i \uparrow}^{\dagger} c_{j \downarrow}^{\dagger}-c_{i \downarrow}^{\dagger} c_{j \uparrow}^{\dagger}+\text { H.c. }\right), \\
H_{\mu} & =-\mu \sum_{i} n_{i},
\end{aligned}
$$

where $t$ is the hopping parameter, $\Delta_{i j}$ is the pairing amplitude, $\mu$ is the chemical potential, and $\sigma$ denotes spin up, $\uparrow$, and down, $\downarrow$, respectively. For the $d$-wave pairing symmetry, $\Delta_{i j}= \pm \Delta$ for the $x$ and $y$ directions, respectively. For the $s$-wave pairing symmetry, $\Delta_{i j}=\Delta$ for both directions. The particle number operator $n_{i}$ is given by $n_{i}=\sum_{\sigma} c_{i \sigma}^{\dagger} c_{i \sigma}$.
Meanwhile, the $t-J$ Hamiltonian can be written as follows:

$$
H_{t-J}=\mathcal{P}_{\mathrm{G}}\left(H_{t}+H_{J}\right) \mathcal{P}_{\mathrm{G}},
$$

where $H_{t}$ is the same as in Eq. (10), and

$$
H_{J}=J \sum_{\langle i, j\rangle}\left(\mathbf{S}_{i} \cdot \mathbf{S}_{j}-n_{i} n_{j} / 4\right),
$$

where $J$ is the spin exchange coupling constant with $J>0$ [31-33]. Note that, unless mentioned otherwise, all the energy scales are measured in units of $t$ throughout this work.

Similar to what is done for the $5 / 2$ state problem, we would like to compute the overlap between the exact ground states of the $t-J$ model and the projected BCS theory. To do so, there are three important preliminary works.

First, it is important to check the symmetries of the $t-J$ and projected BCS Hamiltonians, which can crucially affect the overlap. Details of the group theoretical analysis are provided in Appendix B. Second, we confirm if there is any parameter regime, where the two Hamiltonians are guaranteed to be closely connected. To this end, it is proved in Sec. II C that the two Hamiltonians are entirely equivalent at half filling. Third, the overlap should be properly defined in the presence of particle number fluctuations. The precise definition of the proper overlap is provided in Sec. II D.

\section{Equivalence at half filling}

The equivalence between the $t-J$ and projected BCS Hamiltonians at half filling can be proved analytically. With details of the proof presented in Appendix C, here, we provide a brief summary.

We begin by decomposing the Schrödinger equation of the projected BCS theory into a system of simultaneous equations connecting between different particle number sectors. Then, we take the limit of large $\mu$ to maximize the number of electrons under the no-double-occupancy constraint, which leads to half filling. In this limit, we carefully expand the weighting amplitude of the energy eigenstate in each particle number sector in orders of $1 / \mu$. Finally, by keeping only the most dominant terms, it is proved that the projected BCS theory becomes entirely equivalent to the Heisenberg model, i.e., the $t-J$ model at half filling.

Several aspects of the proof are worthwhile to mention. First, the equivalence is not just for the ground state, but rather the entire energy eigenstates at half filling. Second, the proof works regardless of the pairing symmetry or the lattice structure. As shown by explicit computations, the overlap is exactly unity at half filling not only for the $d$-wave pairing symmetry, but also for the $s$-wave pairing symmetry in the square lattice. The same is true for the triangular lattice. Third, the equivalence at half filling does not necessarily guarantee a high overlap upon doping. The overlap at finite doping depends crucially on the pairing symmetry and the lattice structure. It is shown that, in the square lattice, the overlap can remain high with finite $\Delta$ for the $d$-wave pairing symmetry, while not for the $s$-wave counterpart. The overlap is generally quite low at moderate doping in the triangular lattice regardless of the pairing symmetry.

To explicitly compute the overlap between the exact ground states of the $t-J$ model and the projected BCS theory, 


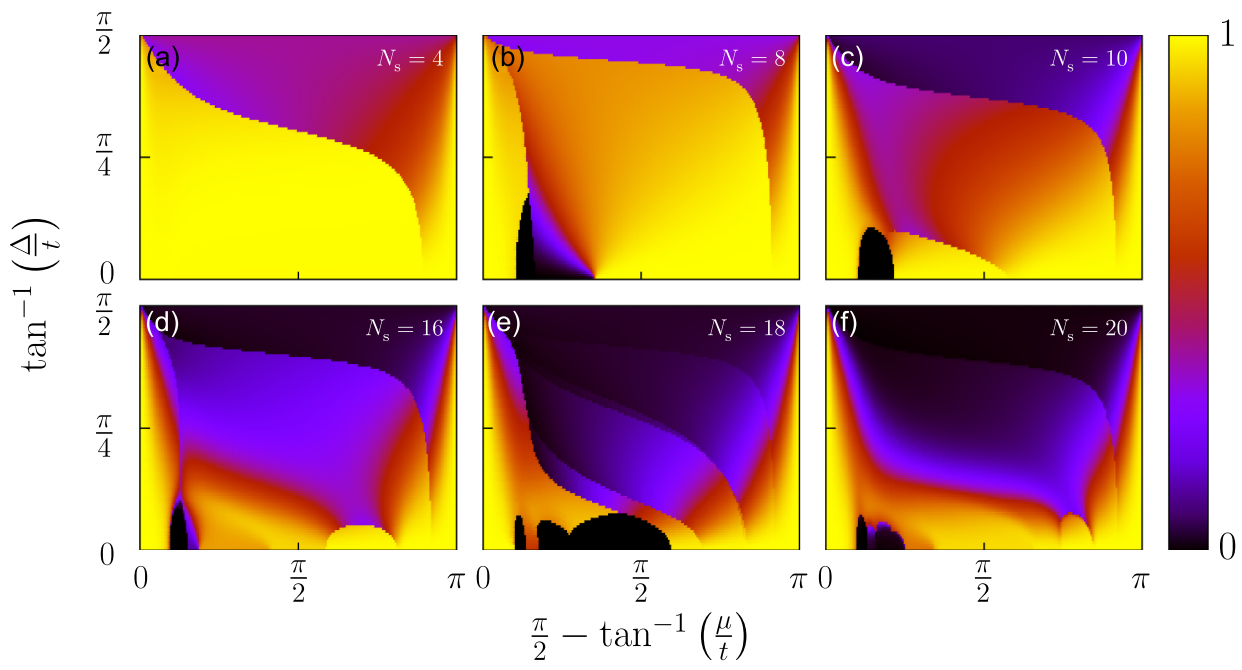

FIG. 1. Map of the number-weighted overlap squared (NWOS) between the exact ground states of the $t$ - $J$ model and the projected BCS theory with the $d$-wave pairing symmetry in the square lattice. Here, the spin exchange coupling constant of the $t-J$ model is set to be $J / t=0.5$. Denoted in color with bright yellow being unity and black being zero, the NWOS is computed as a function of pairing amplitude $\Delta$ and chemical potential $\mu$. As one can see, the NWOS is exactly unity regardless of $\Delta$ at sufficiently large $\mu$, proving that the Néel state is precisely captured by the projected BCS theory at half filling. Note that the NWOS also becomes unity for sufficiently large negative $\mu$, i.e., near the vacuum, where both spin exchange and pairing terms play equally negligible roles. The number of sites is varied as $N_{s}=4,8,10,16,18$, and 20 .

below, we define the proper overlap valid in the presence of particle number fluctuations.

\section{Number-weighted overlap squared}

The exact ground state of the projected BCS theory $\left|\psi_{\mathrm{PBCS}}\right\rangle$ can be expanded in terms of its number-projected component in each particle number sector $\left|\phi_{h}^{\mathrm{PBCS}}\right\rangle$ as follows:

$$
\left|\psi_{\mathrm{PBCS}}\right\rangle=\sum_{h=0}^{N_{s}} \lambda_{h}\left|\phi_{h}^{\mathrm{PBCS}}\right\rangle,
$$

where $h$ is the number of holes, related with that of sites, $N_{s}$, and that of electrons, $N_{e}$, via $h=N_{s}-N_{e}$. Note that the RVB state can be also similarly expanded.

Meanwhile, any exact ground state of the $t-J$ model has a fixed number of particles. This means that each particle number sector has its own exact ground state of the $t-J$ model. A pressing question is how to compute the overlap between $\left|\psi_{\mathrm{PBCS}}\right\rangle$ and the set of the exact ground states of the $t$ - $J$ model, $\left\{\left|\phi_{h}^{t-J}\right\rangle\right\}$.

The traditional method is to project $\left|\psi_{\mathrm{PBCS}}\right\rangle$ onto the specific desired particle number sector and compute its overlap with $\left|\phi_{h}^{t-J}\right\rangle$ in that sector, i.e.,

$$
\mathcal{O}_{h}^{2}=\left|\left\langle\phi_{h}^{\mathrm{PBCS}} \mid \phi_{h}^{t-J}\right\rangle\right|^{2},
$$

which we call the number-projected overlap squared (NPOS). As shown previously $[13,14]$, the NPOS provides a reasonable measure of the overlap. Unfortunately, however, there is a rather large degree of arbitrariness in this method, causing critical finite-size effects. That is, the particle number is fixed by two separate processes: (i) projecting $\left|\psi_{\mathrm{PBCS}}\right\rangle$ onto the specific, desired particle number sector and (ii) choosing the chemical potential in $H_{\mathrm{PBCS}}$. Applied simultaneously, these two processes are often inconsistent with each other, not to mention redundant.
We fix this problem by devising the proper overlap valid in the presence of particle number fluctuations, which is defined as the weighted NPOS over all possible particle number sectors:

$$
\overline{\mathcal{O}^{2}}=\sum_{h}\left|\lambda_{h}\right|^{2} \mathcal{O}_{h}^{2},
$$

which we call the number-weighted overlap squared (NWOS). Note that the weighting factor can be computed via $\left|\lambda_{h}\right|^{2}=$ $\left\langle\psi_{\text {PBCS }}\left|\mathcal{P}_{h}\right| \psi_{\text {PBCS }}\right\rangle$, where $\mathcal{P}_{h}$ is the number projection operator.

To appreciate the actual implementation of the NWOS, it is instructive to consider the $2 \times 2$ system, where the NWOS can be computed analytically. See Appendix D for details. Below, we present the numerical analysis of the NWOS via fullfledged exact diagonalization in the $2 \times 2$ and larger systems.

\section{E. Overlap map}

Figure 1 shows the map of the NWOS between the exact ground states of the $t-J$ model and the projected BCS theory with the $d$-wave pairing symmetry in the square lattice. Here, the NWOS is computed as a function of $\Delta$ and $\mu$, both of which are varied for all possible values. Note that $N_{s}$ is appropriately chosen for the proper tessellation of the square lattice preserving the rotational symmetry. See Appendix E for details.

There are several important features to be noted. First, as expected from the equivalence at half filling, the NWOS is unity regardless of $\Delta$ in the limit of large $\mu$. This confirms that the long-range antiferromagnetic order is precisely captured by the projected BCS theory. Note that, strictly speaking, one should include a staggered magnetic field in the projected BCS Hamiltonian to generate the true longrange antiferromagnetic order. However, we do not need to do so here since it is now a well-established fact that the true long-range antiferromagnetic order emerges in the 


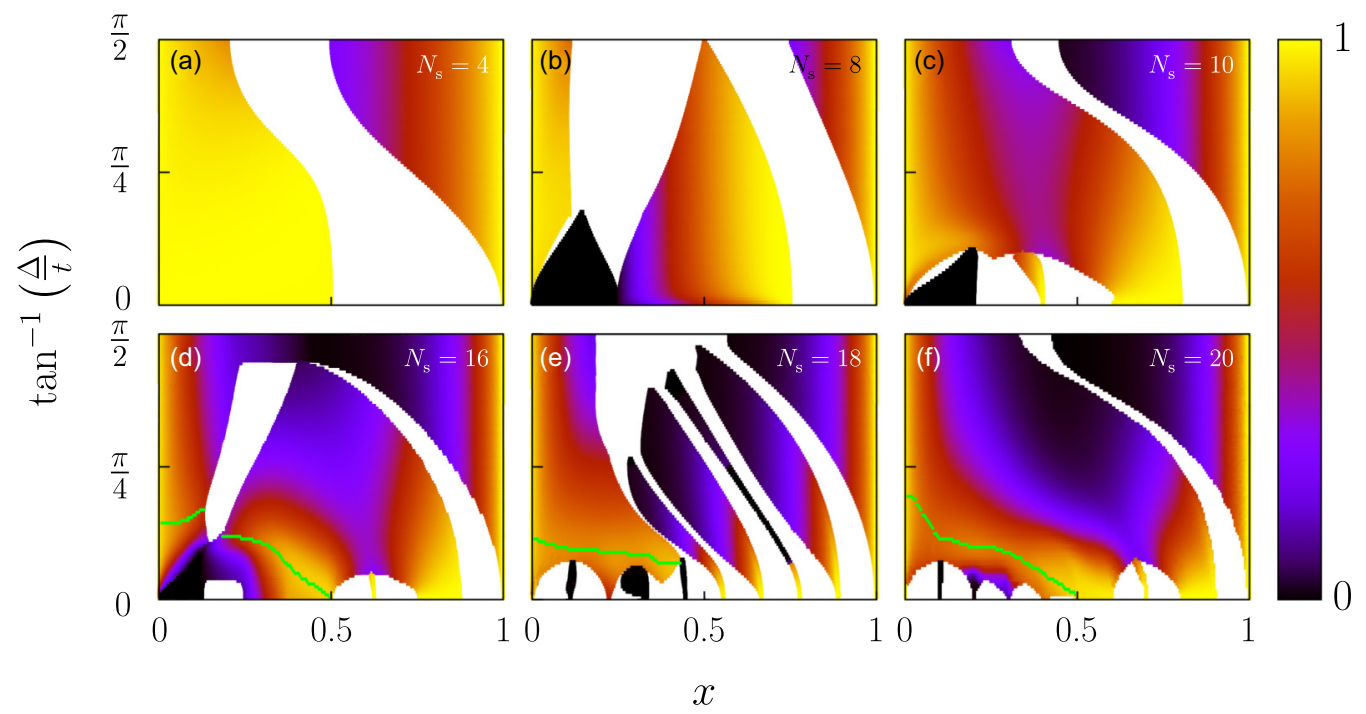

FIG. 2. Similar map of the NWOS to Fig. 1 with the abscissa converted to the hole concentration $x$. The chemical potential $\mu$ is related with $x$ via $x=1-\sum_{i}\left\langle n_{i}\right\rangle / N_{s}$. The optimal pairing amplitude producing the maximum NWOS, $\Delta_{\max }$, is denoted by the green lines in [(d)-(f)] as a function of $x$. Note that the NWOS is exactly unity regardless of $\Delta$ at $x=0$, i.e., half filling, and $x=1$, i.e., the vacuum. The white, or vacant regions indicate that the exact ground state of the projected BCS theory is not available there.

antiferromagnetic Heisenberg model [34-39]. As proven numerically here and analytically in Appendix C, the projected BCS Hamiltonian is exactly identical to the antiferromagnetic Heisenberg model at half filling.

Second, the NWOS also becomes unity along the line of $\Delta=0$ for sufficiently large negative $\mu$, indicating that there is no pairing in the weakly correlated regime near the vacuum. Here, the exact ground states of the $t-J$ model and the projected BCS theory become identical since both spin exchange and pairing terms play equally negligible roles. In this regime, the ground state simply becomes the usual Fermi liquid.

Third, most importantly, the NWOS can remain quite high (typically $\gtrsim 80 \%-90 \%$ ) along the well-defined curve of nonzero $\Delta$ as a function of $\mu$, especially prominent at $N_{s}=16$ and 20. Note that, at $N_{s}=18$, there is a large black dome around $\mu=0$, masking the otherwise high NWOS region. The high NWOS can be obtained in this region if the NWOS is computed by using the first excited state of the projected BCS theory, which is nearly degenerate with the exact ground state.

Note that the spin exchange coupling constant of the $t-J$ model is set to be $J / t=0.5$ in Fig. 1. It is shown in further analyses of the NWOS that the optimal pairing amplitude grows as $J / t$ increases. See Appendix $\mathrm{F}$ for the detailed behavior of the NWOS with variation of $J / t$.

Actually, it is physically more meaningful to express the overlap map as a function of hole concentration $x$ rather than $\mu$. To this end, we convert $\mu$ to $x$ by inverting the relationship $x=1-\sum_{i}\left\langle n_{i}\right\rangle / N_{s}$, where $\left\langle n_{i}\right\rangle$ depends implicitly on $\mu$.

Figure 2 shows the map of the NWOS between the exact ground states of the $t-J$ model and the projected BCS theory as a function of $x$ and $\Delta$, from which one can determine the optimal pairing amplitude, $\Delta_{\max }$, producing the maximum NWOS at each value of $x$. As one can see from the green lines in (d)-(f) in Fig. 2, $\Delta_{\max }$ is clearly lifted from zero, indicating that there is a well-defined pairing correlation up to $x \simeq 0.5$.
At this point, it is worthwhile to discuss the significance of the overlap. To begin with, the NWOS is the weighted square of overlap, and therefore its square root is a more appropriate measure for the overlap than the NWOS itself. In this sense, the overlap can be regarded as being more than $90 \%$ along the lines of $\Delta_{\max }$ in (d)-(f) in Fig. 2. Given this value, the significance of the overlap can be appreciated by comparing it with those obtained in other well-established examples of strongly correlated electron systems.

One example is the FQHE states at the Jain sequence, which include not only the celebrated Laughlin state, but also general CF states. In this case, the overlap is typically around $99 \%$ in finite-size systems with particle number ranging from $N=6$ to 12 [17], which is sufficiently high so that there is little doubt for the validity of the Laughlin/CF states.

However, the situation is not always this clear. As previously mentioned, the even-denominator FQHE state at filling factor $v=5 / 2$ is believed to be a paired quantum Hall state, for which one of the most promising candidate trial states is the MR Pfaffian state. The overlap between the exact $5 / 2$ and MR Pfaffian states is typically less than $60 \%-80 \%$ in finite-size systems with $N=10-12$ for the pure Coulomb interaction [28-30]. The situation is actually even worse since the overlap can become completely zero, depending on geometric details of the finite-size system. This is due to the fact that the pure Coulomb interaction is essentially the critical point dividing two different phases with one captured by the MR Pfaffian state and the other by some unknown state. Fortunately, the situation can be improved in favor of the MR Pfaffian state if one tweaks the interaction by tuning the Haldane pseudopotential or including the effect of finite thickness, in which case the overlap can increase up to $90 \%$ [28-30].

Another example is the factional Chern insulator (FCI) occurring in the $1 / 3$-filled Chern flat band, which is envisioned as the lattice analog of the Laughlin state. A good trial state for the 1/3-filled FCI can be constructed by importing 
the amplitudes of the Laughlin state and attaching them to appropriately chosen basis states in the Chern flat band, called the hybrid localized Wannier states [40]. It is shown that the overlap between this trial state and the exact 1/3-filled FCI state is scattered over a wide range from 80 to $99 \%$ in finitesize systems with $N=6-8$, depending on lattice structures of the Chern flat band.

In summary, based on the comparison with these wellestablished examples, the overlap of $90 \%$ in finite-size systems with roughly ten particles can be taken as a solid piece of evidence supporting the validity of the trial state.

It is emphasized again that $\Delta_{\max }$ is clearly lifted from zero as shown in (d)-(f) in Fig. 2, which is strongly supportive of the existence of superconductivity. Unfortunately, however, the actual detailed shape of $\Delta_{\max }$ as a function of $x$ suffers from somewhat considerable finite-size effects. In the next section, we show that finite-size effects can be significantly alleviated by using the twisted boundary condition.

\section{F. Twisted boundary condition}

The twisted boundary condition is actually equivalent to the periodic boundary condition under the appropriate gauge transformation implementing a flux insertion. For simplicity, let us consider the rectangular system with $N_{x} \times N_{y}$ sites, in which case the appropriate gauge transformation is given as follows:

with

$$
c_{j \sigma}^{\dagger} \rightarrow e^{i \phi_{j \sigma}} c_{j \sigma}^{\dagger},
$$

$$
\phi_{j \sigma}=\mathcal{A}_{\sigma} \cdot \mathbf{r}_{j},
$$

where $\mathcal{A}_{\sigma}$ is the vector potential implementing the flux insertion, which can be in general spin-dependent. As shown below, we consider the spin-dependent vector potential to preserve the translational symmetry in the presence of the pairing term.

Note that the pairing term in the projected BCS Hamiltonian explicitly breaks the gauge symmetry, and therefore the flux cannot be inserted arbitrarily. In our current situation, a usual implementation of the twisted boundary condition, i.e., assigning the same phase twist for different spins, results in breaking the translational symmetry. To overcome this problem, one needs to assign opposite phase twists for different spins. Specifically, we choose the following vector potential:

$$
\mathcal{A}_{\sigma}=\operatorname{sgn}(\sigma)\left(\frac{\theta_{x}}{N_{x}}, \frac{\theta_{y}}{N_{y}}\right),
$$

where $\operatorname{sgn}(\sigma)= \pm 1$ for $\sigma=\uparrow$ and $\downarrow$, respectively, and $\theta_{x}$ and $\theta_{y}$ are the twist angles across the system boundary along the $x$ and $y$ directions, respectively.

To confirm that this choice of the spin-dependent vector potential preserves the translational symmetry, let us see what happens to the BCS Hamiltonian after the above gauge transformation:

$$
\begin{aligned}
H_{t} & =-t \sum_{\langle j, k\rangle, \sigma}\left[e^{i\left(\phi_{j \sigma}-\phi_{k \sigma}\right)} c_{j \sigma}^{\dagger} c_{k \sigma}+\text { H.c. }\right], \\
H_{\Delta} & =\sum_{\langle j, k\rangle} \Delta_{j k}\left[e^{i\left(\phi_{j \uparrow}+\phi_{k \downarrow}\right)} c_{j \uparrow}^{\dagger} c_{k \downarrow}^{\dagger}-e^{i\left(\phi_{j \downarrow}+\phi_{k \uparrow}\right)} c_{j \downarrow}^{\dagger} c_{k \uparrow}^{\dagger}+\text { H.c. }\right],
\end{aligned}
$$

while $H_{\mu}$ remains the same as before. Note that the acquired phase in the hopping term depends only on the relative distance, i.e., $\phi_{j \sigma}-\phi_{k \sigma}=\mathcal{A}_{\sigma} \cdot\left(\mathbf{r}_{j}-\mathbf{r}_{k}\right)$. This means that the hopping term preserves the translational symmetry. Meanwhile, the pairing term can preserve the translational symmetry if the phase twist is opposite for different spins, i.e., $\phi_{j \uparrow}+\phi_{k \downarrow}=-\left(\phi_{j \downarrow}+\phi_{k \uparrow}\right)=\mathcal{A}_{\uparrow} \cdot\left(\mathbf{r}_{j}-\mathbf{r}_{k}\right)$.

Actually, one should also check how $H_{J}$ is gaugetransformed. To this end, it is convenient to rewrite $H_{J}$ in terms of electron creation and annihilation operators as follows:

$$
\begin{array}{r}
H_{J}=\frac{J}{2} \sum_{\langle j, k\rangle}\left[c_{j \uparrow}^{\dagger} c_{k \downarrow}^{\dagger} c_{j \uparrow} c_{k \downarrow}+c_{j \downarrow}^{\dagger} c_{k \uparrow}^{\dagger} c_{j \downarrow} c_{k \uparrow}\right. \\
\left.-c_{j \uparrow}^{\dagger} c_{k \downarrow}^{\dagger} c_{j \downarrow} c_{k \uparrow}-c_{j \downarrow}^{\dagger} c_{k \uparrow}^{\dagger} c_{j \uparrow} c_{k \downarrow}\right],
\end{array}
$$

where the first and last two terms constitute the direct and exchange parts of $H_{J}$, respectively. While the direct part is simply invariant under the gauge transformation, the exchange part is gauge-transformed as follows:

$$
\begin{aligned}
& H_{J}^{\mathrm{ex}}=-\frac{J}{2} \sum_{\langle j, k\rangle} {\left[e^{i\left(\phi_{j \uparrow}+\phi_{k \downarrow}-\phi_{j \downarrow}-\phi_{k \uparrow}\right)} c_{j \uparrow}^{\dagger} c_{k \downarrow}^{\dagger} c_{j \downarrow} c_{k \uparrow}\right.} \\
&\left.+e^{i\left(\phi_{j \downarrow}+\phi_{k \uparrow}-\phi_{j \uparrow}-\phi_{k \downarrow}\right)} c_{j \downarrow}^{\dagger} c_{k \uparrow}^{\dagger} c_{j \uparrow} c_{k \downarrow}\right],
\end{aligned}
$$

which can preserve the translational symmetry since, as before, the acquired phase depends only on the relative distance, i.e., $\phi_{j \uparrow}+\phi_{k \downarrow}-\phi_{j \downarrow}-\phi_{k \uparrow}=2 \mathcal{A}_{\uparrow} \cdot\left(\mathbf{r}_{j}-\mathbf{r}_{k}\right)$.

In addition to the translational symmetry, one can also preserve the reflection symmetry by appropriately coordinating $\theta_{x}$ and $\theta_{y}$. Specifically, for the square system with $N_{x}=N_{y}$, the reflection symmetry can be preserved if one sets $\theta_{x}$ and $\theta_{y}$ to be the same, say $\theta$. In this work, we focus on the $N_{s}=16$ system with $N_{x}=N_{y}=4$, where the NWOS can be computed for various different values of $\theta$ within a reasonable computing time.

Figure 3(a) shows the map of the NWOS under the twisted boundary condition as a function of $\theta=\theta_{n}=2 \pi n / N_{\text {grid }}$ with $n=0, \ldots, N_{\text {grid }} / 2$ and $N_{\text {grid }}=10$. Note that the NWOS has a mirror symmetry with respect to $\theta$ inverted across $\pi$. As one can see, the NWOS map remains overall similar regardless of $\theta$, while detailed features are somewhat different. Interestingly, some maps of the NWOS under the twisted boundary condition at $N_{s}=16$ resemble those under the periodic boundary condition at larger system sizes, $N_{s}=18$ and 20. To be specific, the NWOS maps at $\theta=\pi$ and $3 \pi / 5$ resemble those of the $N_{s}=18$ [Fig. 2(e)] and 20 [Fig. 2(f)] system, respectively. This suggests a possibility that finite-size effects can be alleviated by taking an average of the NWOS over twist angles.

Figure 3(b) shows the map of the averaged NWOS over twist angles of $\theta=\theta_{n}$ :

$$
\overline{\mathcal{O}}_{\text {avg }}=\frac{1}{N_{\text {grid }}} \sum_{n=0}^{N_{\text {grid }}-1} \overline{\mathcal{O}^{2}}\left(\theta_{n}\right),
$$

where $\overline{\mathcal{O}^{2}}\left(\theta_{n}\right)$ is the NWOS at $\theta_{n}$. Note that the averaged NWOS can be in principle computed for each individual point in the $x-\Delta$ phase space. Unfortunately, however, there are white regions in the phase space, where the NWOS is not 

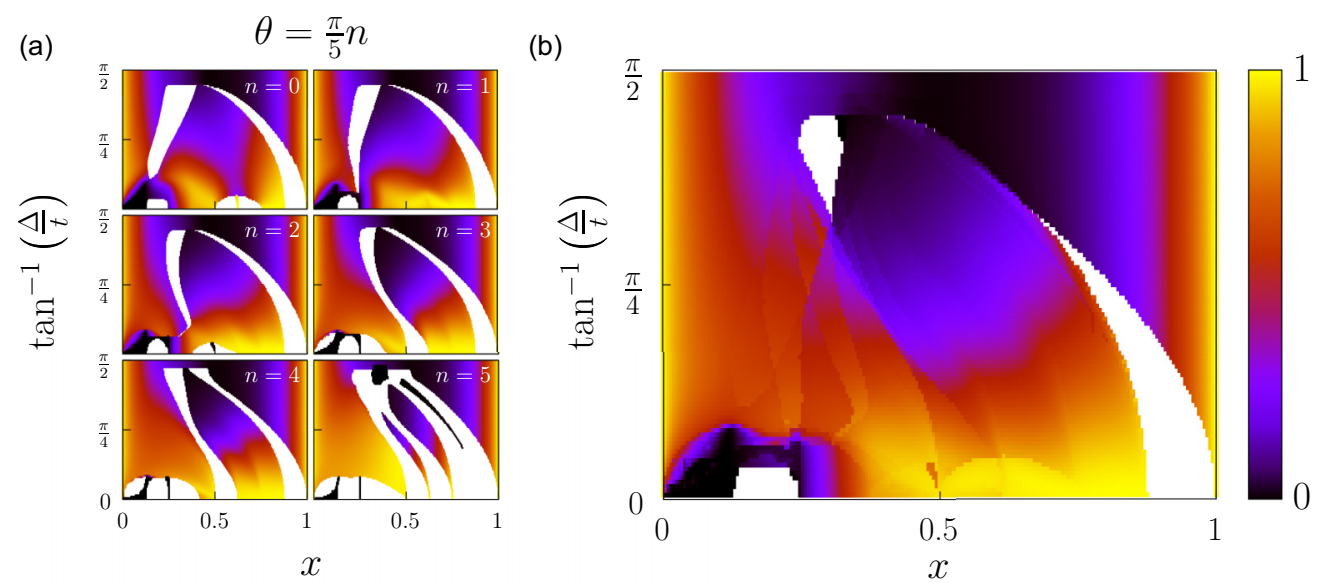

FIG. 3. Map of the NWOS under the twisted boundary condition. (a) Map of the NWOS as a function of the twist angle, $\theta=\frac{\pi}{5} n$, with $n=0, \ldots, 5$ between the exact ground states of the $t-J$ model and the projected BCS theory with the $d$-wave pairing symmetry in the square lattice with $N_{s}=16$. (b) Map of the averaged NWOS over twist angles of $\theta=\frac{\pi}{5} n$ with $n=0, \ldots, 9$. Note that the NWOS is periodic as a function of $\theta$ with the period of $2 \pi$, while having the mirror symmetry with respect to $\theta$ inverted across $\pi$.

available at some twist angles. Such missing twist angles are skipped in the averaging process.

The map of the averaged NWOS shows a rather wellconverged pattern, which is quite resemblant of the NWOS map in Fig. 2(f) for the $N_{s}=20$ system under the periodic boundary condition. This demonstrates that finite-size effects can be indeed significantly alleviated by using the twisted boundary condition.

Now, one may wonder if the existence of nonzero $\Delta_{\max }$ actually means true superconductivity. To answer this question, below, we perform the direct computation of superconducting order parameters in the projected BCS theory with $\Delta_{\max }$ used as an input parameter for the bare pairing amplitude.

\section{G. Superconducting order parameter}

One of the greatest advantages of the projected BCS theory is that, by utilizing the intrinsic nature of the superconducting ground state containing particle number fluctuations, one can directly compute the superconducting order parameter, $\left\langle c_{i \uparrow} c_{j \downarrow}\right\rangle$, instead of taking the large-distance limit of the off-diagonal long-range order (ODLRO), $\left\langle c_{i \uparrow} c_{j \downarrow} c_{k \downarrow}^{\dagger} c_{l \uparrow}^{\dagger}\right\rangle$. Note that $i(k)$ and $j(l)$ denote the coordinates of nearestneighboring sites with $i$ and $k$ largely separated.

Specifically, the superconducting order parameter can be computed as follows. First, $\Delta_{\max }$ is determined by tracing the maximum NWOS as a function of $x$ as shown in Fig. 4(a). Then, we compute $\left\langle c_{i \uparrow} c_{j \downarrow}\right\rangle$ by simply taking the expectation value of $c_{i \uparrow} c_{j \downarrow}$ for the exact ground states of the projected BCS theory with $\Delta_{\max }$ used as an input parameter for the pairing amplitude. Figure 4(b) shows the resulting superconducting order parameter. It is important to note that the superconducting order parameter vanishes as $x$ decreases, while $\Delta_{\max }$ remains finite. This split between the pairing amplitude and the superconducting order parameter is due to the strong correlation between Cooper pairs.

To elucidate the role of the strong correlation more clearly, we compare $\left\langle c_{i \uparrow} c_{j \downarrow}\right\rangle$ with the "bare" superconducting order parameter $\left\langle c_{i \uparrow} c_{j \downarrow}\right\rangle_{0}$, which would be the superconducting or- der parameter if there were no Gutzwiller projection:

$$
\left\langle c_{i \uparrow} c_{j \downarrow}\right\rangle_{0}=\sum_{\mathbf{k}} \frac{\Delta_{0, \mathbf{k}}}{2 E_{0, \mathbf{k}}} \cos k_{x},
$$

where $E_{0, \mathbf{k}}=\sqrt{\xi_{0, \mathbf{k}}^{2}+\Delta_{0, \mathbf{k}}^{2}}$ with $\Delta_{0, \mathbf{k}}=2 \Delta_{\max }\left(\cos k_{x}-\right.$ $\left.\cos k_{y}\right)$ and $\xi_{0, \mathbf{k}}=-2 t\left(\cos k_{x}+\cos k_{y}\right)-\mu_{0}$. Note that $\mu_{0}$ is determined from the condition that the "bare" hole concentration is the same as $x: \frac{1}{N_{s}} \sum_{\mathbf{k}} \xi_{0, \mathbf{k}} / E_{0, \mathbf{k}}=x$. As shown in Fig. 4(b), the bare superconducting order parameter does not vanish at low doping similar to $\Delta_{\max }$.

While important to quantify the strength of superconductivity, the superconducting order parameter is not a physical observable by itself. In this context, a relevant physical observable is the expectation value of the pairing energy, $\Delta_{\max }\left\langle c_{i \uparrow} c_{j \downarrow}\right\rangle$. Figure 4(c) shows that, similar to the real versus bare superconducting order parameter, $\Delta_{\max }\left\langle c_{i \uparrow} c_{j \downarrow}\right\rangle$ vanishes at low doping while its bare counterpart, $\Delta_{\max }\left\langle c_{i \uparrow} c_{j \downarrow}\right\rangle_{0}$, does not. It is interesting to mention that the similar split between the bare pairing amplitude and the real superconducting order parameter was previously observed [5,7].

\section{H. Comparison with the RVB state}

To put the results of the projected BCS theory into prospective, we also perform the numerical analysis of the NWOS for the RVB state.

To this end, it is necessary to construct the RVB state tailormade for each specific finite system. Such a construction can be accomplished by finding the amplitude of the RVB state for a given basis state with spin up and down electrons located in the specific configuration, $\left\{\mathbf{r}_{\uparrow}, \mathbf{r}_{\downarrow}\right\}$, while satisfying the nodouble-occupancy condition:

$$
\psi_{\mathrm{RVB}}\left(\left\{\mathbf{r}_{\uparrow}, \mathbf{r}_{\downarrow}\right\}\right)=\operatorname{det}\left(\tilde{g}_{i j}\right),
$$

where $\tilde{g}_{i j}=\tilde{g}\left(\mathbf{r}_{i}-\mathbf{r}_{j}\right)$ is the Fourier transform of $g_{\mathbf{k}}=$ $\Delta_{\mathbf{k}} /\left(\xi_{\mathbf{k}}+E_{\mathbf{k}}\right) \quad$ with $\quad E_{\mathbf{k}}=\sqrt{\xi_{\mathbf{k}}^{2}+\Delta_{\mathbf{k}}^{2}}, \quad \Delta_{\mathbf{k}}=2 \Delta\left(\cos k_{x}\right.$ $\left.\cos k_{y}\right)+\delta$, and $\xi_{\mathbf{k}}=-2 t\left(\cos k_{x}+\cos k_{y}\right)-\mu$. The indices $i$ and $j$ run though the coordinates of all spin up and down 

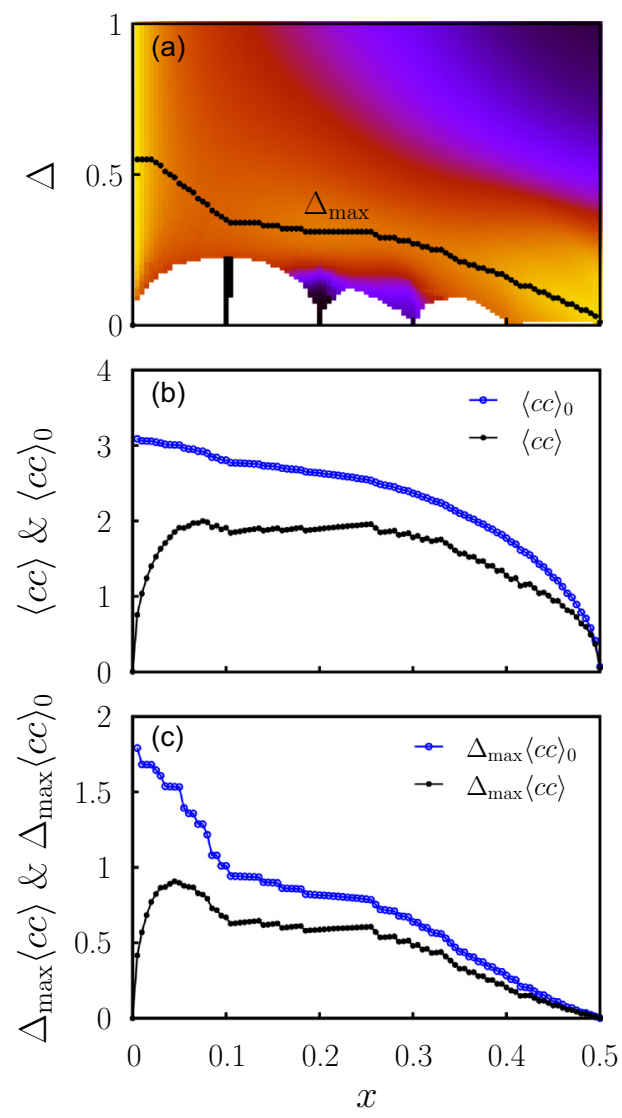

FIG. 4. Optimal pairing amplitude $\Delta_{\max }$ and superconducting order parameter $\langle c c\rangle$ for the projected BCS theory with the $d$-wave pairing symmetry in the square lattice. (a) $\Delta_{\max }$ is plotted as solid circles denoting the locations of the maximum NWOS as a function of $x$. (b) The superconducting order parameter $\langle c c\rangle$ is computed by using the exact ground states of the projected BCS theory with $\Delta_{\max }$ used as an input parameter for the pairing amplitude. $\langle c c\rangle$ is the abbreviation of the superconducting order parameter $\left\langle c_{i \uparrow} c_{j \downarrow}\right\rangle$ with $i$ and $j$ denoting the coordinates of nearest-neighboring sites. Note that the sign of $\left\langle c_{i \uparrow} c_{j \downarrow}\right\rangle$ depends on whether $j$ is the nearest neighbor of $i$ along the $x$ or $y$ direction, as required by the $d$-wave pairing symmetry. Similarly abbreviated, $\langle c c\rangle_{0}$ indicates the "bare" superconducting order parameter. (c) $\Delta_{\max }\langle c c\rangle$ provides the expectation value of the pairing energy with $\Delta_{\max }\langle c c\rangle_{0}$ being its bare counterpart. These results are obtained at $N_{s}=20$.

electrons in $\left\{\mathbf{r}_{\uparrow}, \mathbf{r}_{\downarrow}\right\}$, respectively. Note that a small constant $\delta$ is added to the pairing term $\Delta_{\mathbf{k}}$ for technical convenience. See Appendix A for the detailed derivation of Eq. (24).

Figure 5 shows the map of the NWOS between the exact ground state of the $t-J$ model and the RVB state with the $d$-wave pairing symmetry in the square lattice. Due to its inability to capture the long-range antiferromagnetic order at half filling, the RVB state has zero NWOS with the exact ground state of the $t-J$ model at sufficiently large $\mu$ for $N_{s}=$ $4,10,16$, and 20. For $N_{s}=8$ and 18, the RVB state is not even available beyond certain large $\mu$, where the Gutzwiller projection completely annihilate the BCS state, making the RVB state the null state.

Similar to what is done for the projected BCS theory, the NWOS of the RVB state can be also plotted as a function of $x$ as shown in Fig. 6. Again, the RVB state has zero NWOS with the exact ground state of the $t-J$ model at half filling. Away from half filling, overall, the NWOS of the RVB state shows highly irregular behaviors, strongly depending on $N_{s}$ with broad vacant regions. Even when the NWOS of the RVB state behaves reasonably well at $N_{s}=10$ and 20, the optimal pairing amplitude of the RVB state exhibits quite a different behavior from that of the projected BCS theory at low doping. That is, the optimal pairing amplitude of the RVB state vanishes as $x$ approaches zero, while that of the projected BCS theory remains finite. As explained before, the nonvanishing behavior of the pairing amplitude at low doping is crucial to explain the pseudogap phenomenon.

To make an objective assessment of the correct behavior of the pairing amplitude, we check which state between (i) the exact ground state of the projected BCS theory and (ii) the RVB state has higher overlaps with the exact ground state of the $t-J$ model as a function of $x$. Figure 7 shows the maximum NWOS, $\overline{\mathcal{O}}^{2}$ max , of the projected BCS theory in comparison with that of the RVB state as a function of $x$. As one can see, $\overline{\mathcal{O}}^{2}$ max of the projected BCS theory is pinned to be exactly unity at half filling, while that of the RVB state approaches a seemingly random, smaller value as $x \rightarrow 0$ and drops discontinuously to zero exactly at $x=0$. More importantly, $\overline{\mathcal{O}^{2}}$ max of the projected BCS theory is reduced somewhat upon initial doping but bounces back to high values as $x$ increases. Meanwhile, $\overline{\mathcal{O}^{2}}$ max of the RVB state is generally lower than that of the projected BCS theory except for a narrow range of $x$ at low doping, where the spin liquid phase might occur.

Actually, a close comparison between Figs. 2(f) and 6(f) reveals that the parameter regime, where the RVB state has higher $\overline{\mathcal{O}}^{2}$ max than the exact ground state of the projected BCS theory, falls right into one of the white regions in the overlap map at low doping, where the exact ground states of the projected BCS theory are not available. We believe that, in these regions, the RVB state competes closely against the exact ground state of the projected BCS theory. In other words, there can be two competing states at low to moderate doping with one from the projected BCS theory capturing the pseudogap phase and the other from to the RVB state capturing the spin liquid phase.

In summary, the exact ground states of the projected BCS theory generally provide better trial states for the exact ground states of the $t-J$ model than the RVB state, shedding important light on the pseudogap phenomenon. There is, however, a parameter regime at low to moderate doping, where the exact ground states of the projected BCS theory are not available, while the RVB state provides good overlaps with the exact ground state of the $t-J$ model. Considering that the RVB state can be interpreted as an approximation to the exact ground state of the projected BCS theory, it would be interesting to investigate how the spin liquid phase occurring at this parameter regime can be treated in the grand scheme of the projected BCS theory.

\section{I. $S$-wave pairing symmetry}

Now, we investigate what happens for the projected BCS theory with the $s$-wave pairing symmetry in the square lattice. 


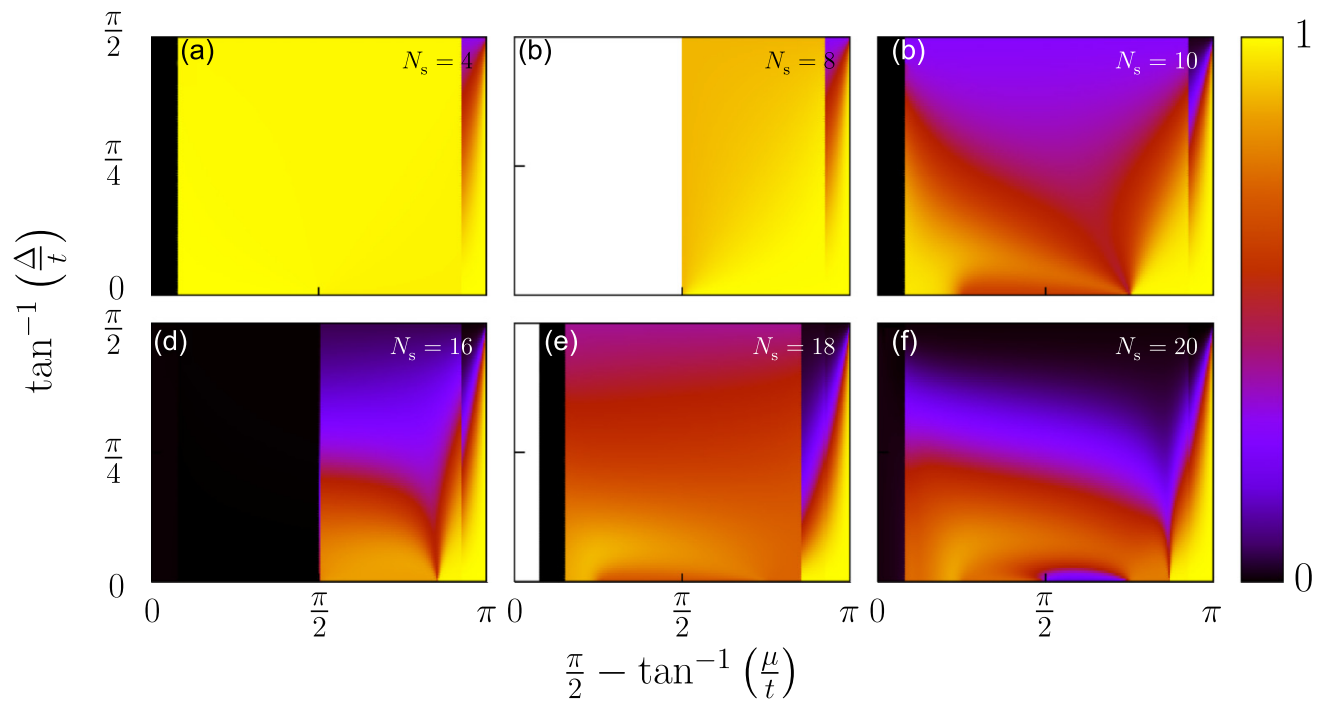

FIG. 5. Map of the NWOS between the exact ground state of the $t$ - $J$ model and the RVB state with the $d$-wave pairing symmetry in the square lattice. Similar to Fig. 1, the spin exchange coupling constant of the $t$ - $J$ model is set to be $J / t=0.5$. The white regions indicate that the RVB state is not available there. Note that the NWOS is entirely zero regardless of $\Delta$ at sufficiently large $\mu$ as expected from the fact that the RVB state cannot capture the Néel state at half filling.

Figure 8 shows that, in this case, the NWOS is always maximized along the line of $\Delta=0$ for all finite $\mu$, indicating that the $s$-wave pairing actually cannot be formed at any finite doping. It is important to note that the maximum NWOS obtained along the line of $\Delta=0$ is always lower than that of the $d$-wave pairing symmetry along the optimal curve of finite $\Delta$ in Fig. 1 since both $s$ - and $d$-wave pairing symmetries generate the same Hamiltonian at $\Delta=0$. In conclusion, the $s$-wave pairing is highly improbable for the $t-J$ model in the square lattice.

\section{J. Geometrical frustration: Triangular lattice}

Now, we investigate the role of the geometrical frustration in the context of the projected BCS theory. Specifically, we perform the NWOS analysis for the projected BCS theory in the triangular lattice.

Historically, the existence of a pairing correlation in the triangular-lattice antiferromagnet has been an important issue ever since the first proposal of the RVB state for hightemperature superconductivity [1]. In fact, the RVB state was proposed by P. W. Anderson as the ground state of the triangular-lattice antiferromagnet prior to the discovery of high-temperature superconductivity [41]. The rationale behind this proposal is that the RVB state can emerge once the long-range antiferromagnetic order is destroyed by the geometrical frustration. Unfortunately, however, the true ground state of the triangular-lattice antiferromagnet turns out to be long-range ordered with the $120^{\circ}$ spin pattern. Despite this

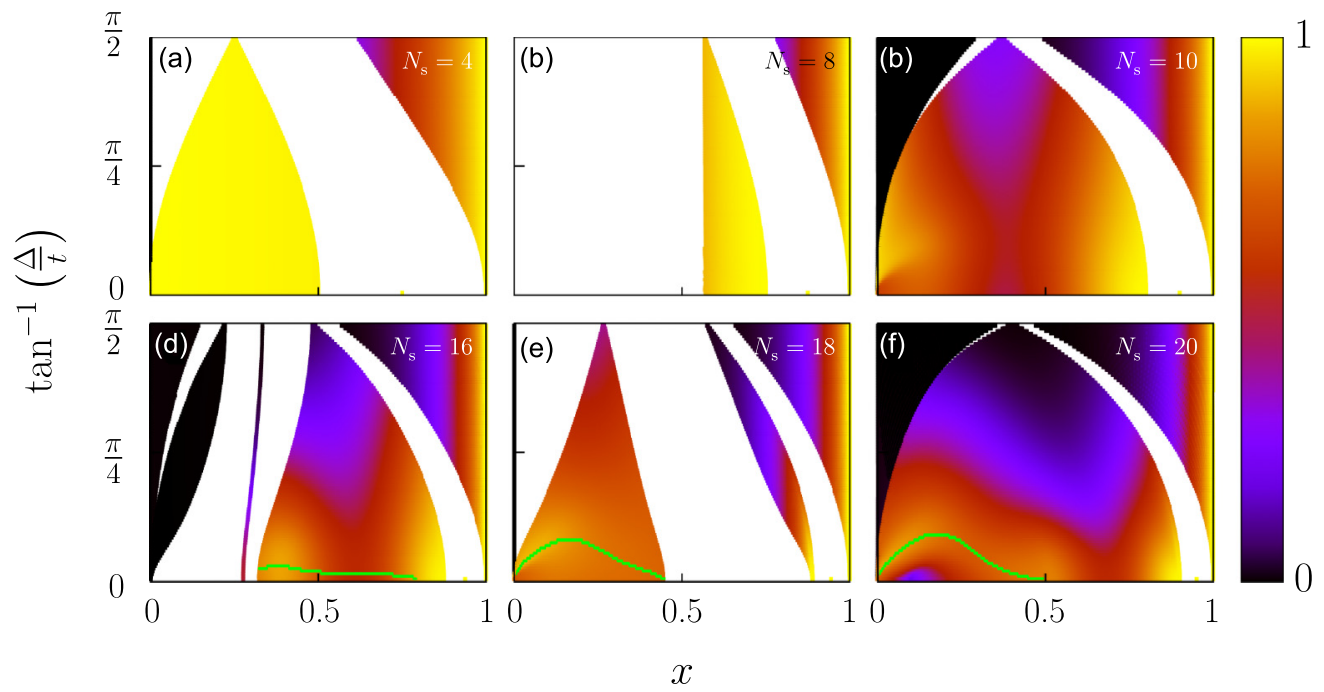

FIG. 6. Similar map of the NWOS to Fig. 5 with the abscissa converted to the hole concentration $x$. As in Fig. $2, \Delta_{\max }$ is denoted by the green lines in (d)-(f) as a function of $x$. The white regions indicate that the RVB state is not available there. Note that the NWOS is entirely zero exactly at half filling. 


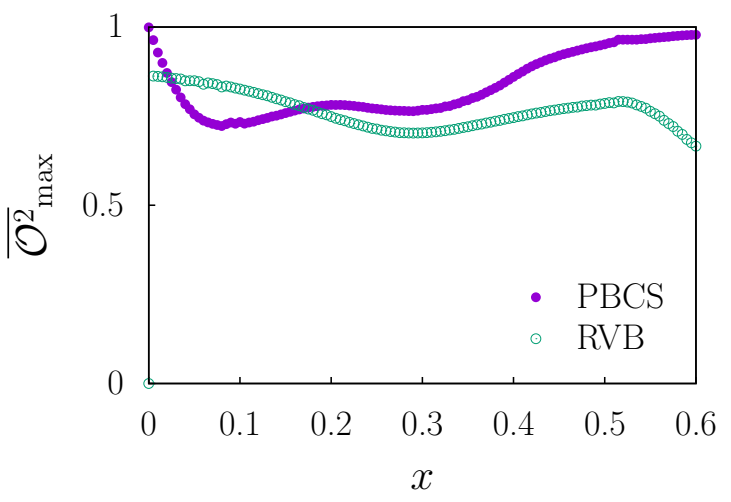

FIG. 7. Maximum NWOS $\overline{\mathcal{O}^{2}}$ max of the projected BCS theory with the exact ground states of the $t-J$ model in comparison with that of the RVB state as a function of $x$. Note that these results are obtained at $N_{s}=20$.

negative result, there might be a possibility that some pairing correlation still emerges with help of the intricate interplay between the geometrical frustration and mobile holes. Here, we check if this is true.

Similar to the square lattice, we consider both $s$ - or $d$-wave pairing symmetries in the triangular lattice. Note that, while simply a constant for the $s$-wave pairing, the pairing amplitude is given as $\Delta_{i j}=\Delta e^{2 i \theta_{i j}}$ for the $d$-wave pairing with $\theta_{i j}$ being the angle between the $x$ axis and the nearest-neighborconnecting vector $\mathbf{r}_{i j}=\mathbf{r}_{i}-\mathbf{r}_{j}$.

Figure 9 shows that, at moderate doping, the projected BCS theory provides poor trial states for the $t-J$ model in the triangular lattice regardless of the pairing symmetry. In conclusion, the geometrical frustration, at least, in the form of the triangular lattice seems to be detrimental to the formation of superconductivity in contrast to the above-mentioned rationale behind the RVB state. This result is consistent with the experimental observation that high-temperature superconduc-

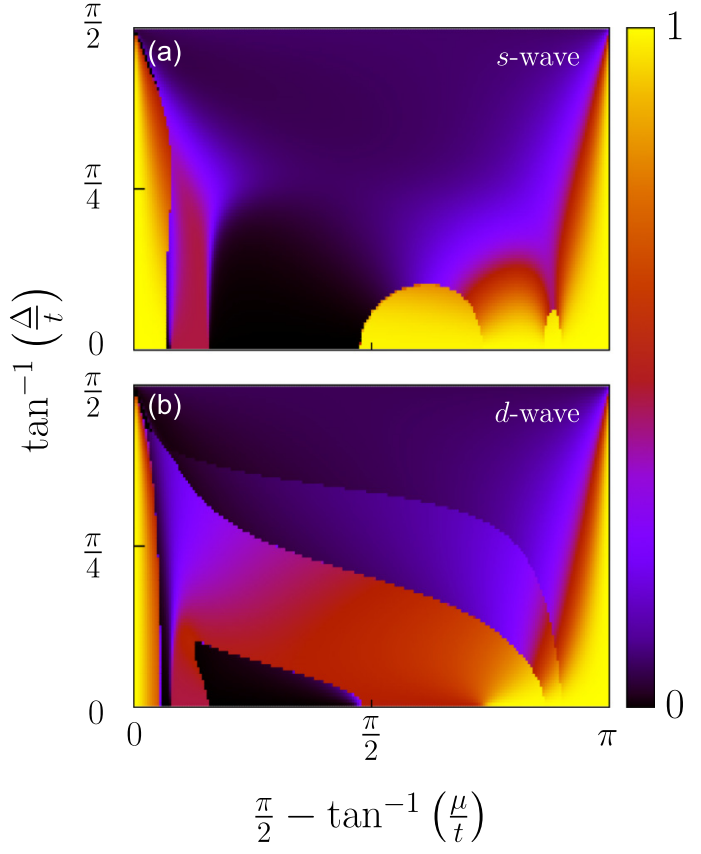

FIG. 9. Map of the NWOS between the exact ground states of the $t-J$ model and the projected BCS theory in the triangular lattice. The pairing symmetries are chosen to be $s$ - and $d$-wave in (a) and (b), respectively. Note that this result is obtained in the $N_{s}=12$ system, which is the only triangular-lattice system accessible via exact diagonalization, satisfying correct symmetries.

tivity occurs almost always in tandem with the Néel state at half filling.

\section{DISCUSSION}

In this work, it is shown that, with the $d$-wave pairing symmetry, the projected BCS theory can provide excellent

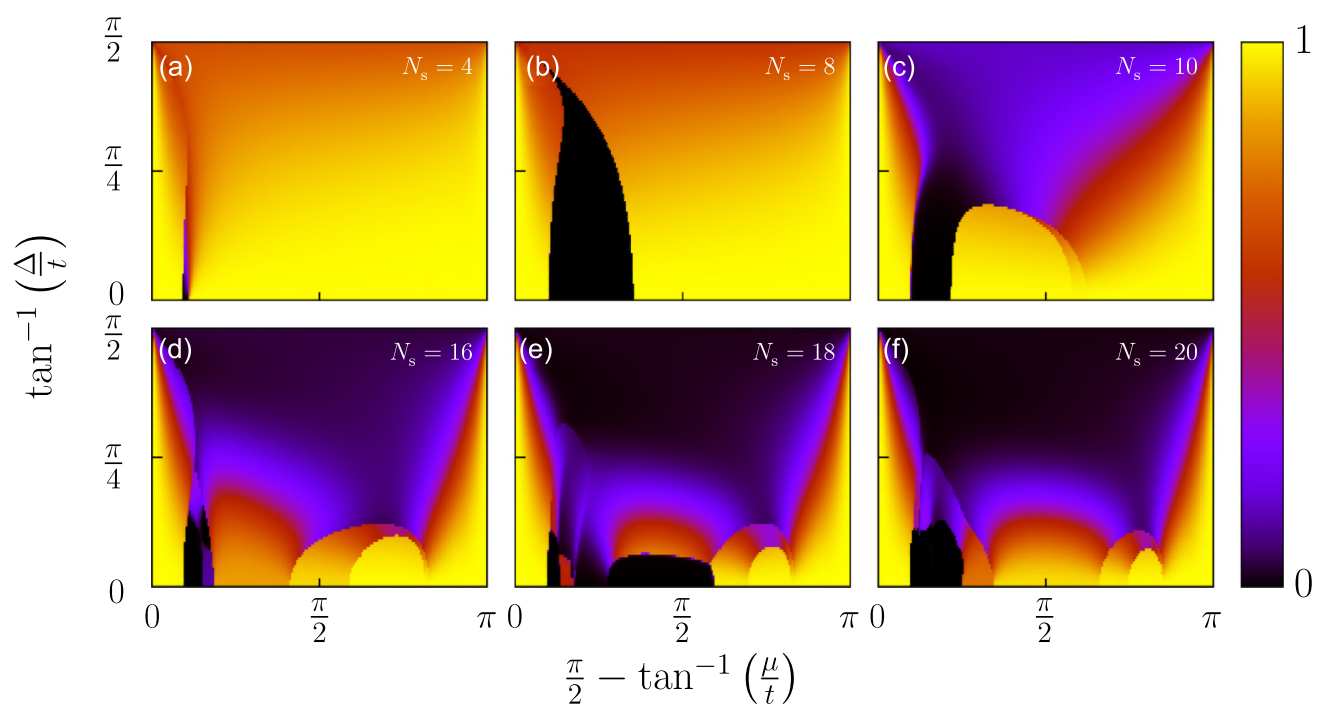

FIG. 8. Similar map of the NWOS to Fig. 1 with the pairing symmetry changed to $s$-wave. As one can see, the NWOS is always maximized along the line of $\Delta=0$ for all finite $\mu$ with a seeming exception at $N_{s}=18$, where the otherwise maximized NWOS is simply masked by the black dome around $\mu=0$. Note that the NWOS is still unity regardless of $\Delta$ in both limits of $\mu=\infty$ and $-\infty$, corresponding to half filling and the vacuum, respectively. 
trial states for the exact ground states of the $t-J$ model in the square lattice for a wide range of hole concentration. As a result, capturing both $\mathrm{AF}$ at half filling and SCSC at moderate doping, a unified theory is obtained by computing the numberweighted overlap squared between the exact ground states of the $t-J$ model and the projected BCS theory with the $d$-wave pairing symmetry.

An important advantage of the projected BCS theory is that it allows direct computation of the superconducting order parameter by utilizing the intrinsic property of superconducting states containing particle number fluctuations. As a result, it is shown that the bare and real superconducting order parameters become split at low doping, which can in principle explain the pseudogap phenomenon.

For future work, it would be interesting to study the spectral properties of the projected BCS theory by computing both normal and anomalous Green's functions. This study is expected to provide an important clue to another great mystery in high-temperature superconductivity, the strange metal behavior. Finally, considering that the RVB state can be interpreted as an approximate ground state of the projected BCS theory, it would be interesting to investigate how the spin liquid/glass phase described by the RVB state can be treated in the grand scheme of the projected BCS theory.

\section{ACKNOWLEDGMENTS}

The authors are grateful to Jainendra K. Jain, Sutirtha Mukherjee, and Yunkyu Bang for insightful discussions. The authors thank Center for Advanced Computation (CAC) at Korea Institute for Advanced Study (KIAS) for providing computing resources for this work. K.P. is supported by a KIAS Individual Grant (PG032303). Also, K.P. acknowledges the hospitality of Subir Sachdev during his sabbatical visit to Harvard University, where parts of this work have been completed.

\section{APPENDIX A: CONSTRUCTION OF THE RVB STATE}

We begin by writing the usual form of the BCS state given in the momentum space as follows:

$$
\begin{aligned}
\left|\psi_{\mathrm{BCS}}\right\rangle & =\prod_{\mathbf{k}}\left(u_{\mathbf{k}}+v_{\mathbf{k}} c_{\mathbf{k} \uparrow}^{\dagger} c_{-\mathbf{k} \downarrow}^{\dagger}\right)|0\rangle \\
& =\mathcal{N} \prod_{\mathbf{k}}\left(1+g_{\mathbf{k}} c_{\mathbf{k} \uparrow}^{\dagger} c_{-\mathbf{k} \downarrow}^{\dagger}\right)|0\rangle \\
& =\mathcal{N} e^{\sum_{\mathbf{k}} g_{\mathbf{k}} c_{\mathbf{k} \uparrow}^{\dagger} c_{-\mathbf{k} \downarrow}^{\dagger}|0\rangle} \\
& =\mathcal{N} e^{\sum_{i, j} \tilde{g}_{i j} c_{i \uparrow}^{\dagger} c_{j \downarrow}^{\dagger}|0\rangle} \\
& =\mathcal{N} \sum_{n} \frac{1}{n !}\left(\sum_{i, j} \tilde{g}_{i j} c_{i \uparrow}^{\dagger} c_{j \downarrow}^{\dagger}\right)^{n}|0\rangle,
\end{aligned}
$$

where $u_{\mathbf{k}}^{2}=\frac{1}{2}\left(1+\xi_{\mathbf{k}} / E_{\mathbf{k}}\right)$ and $v_{\mathbf{k}}^{2}=\frac{1}{2}\left(1-\xi_{\mathbf{k}} / E_{\mathbf{k}}\right)$ with $\xi_{\mathbf{k}}=$ $\epsilon_{\mathbf{k}}-\mu$ and $E_{\mathbf{k}}=\sqrt{\xi_{\mathbf{k}}^{2}+\Delta_{\mathbf{k}}^{2}} \cdot \tilde{g}_{i j}=\tilde{g}\left(\mathbf{r}_{i}-\mathbf{r}_{j}\right)$ is the Fourier transform of $g_{\mathbf{k}}=v_{\mathbf{k}} / u_{\mathbf{k}}$, and $\mathcal{N}$ is the normalization constant. Note that the second line is obtained under the condition that $u_{\mathbf{k}} \neq 0$ for all $\mathbf{k}$. Later, we will come back and address what happens if this condition is violated.
The RVB state can be obtained by applying the Gutzwiller projection operator to the BCS state given in the last line of Eq. (A1):

$$
\left|\psi_{\mathrm{RVB}}\right\rangle=\mathcal{P}_{\mathrm{G}}\left|\psi_{\mathrm{BCS}}\right\rangle=\mathcal{N} \sum_{n} \frac{1}{n !} \mathcal{P}_{\mathrm{G}}\left(\sum_{i, j} \tilde{g}_{i j} c_{i \uparrow}^{\dagger} c_{j \downarrow}^{\dagger}\right)^{n}|0\rangle,
$$

which, unfortunately, is quite complicated since the Gutzwiller projection annihilates the vast majority of terms except for a very few selective ones satisfying the no-double-occupancy condition.

In this situation, instead of specifying each individual surviving term, it is much more convenient to find the amplitude of the RVB state for a given basis state already satisfying the no-double-occupancy condition. For example, consider the basis state, where spin up and down electrons are located in the specific configuration $\left\{\mathbf{r}_{\uparrow}, \mathbf{r}_{\downarrow}\right\}$, where any two coordinates of spin up and down spins are arranged to be different. Then, ignoring the overall normalization constant, the amplitude of the RVB state for this configuration is given as follows:

$$
\psi_{\mathrm{RVB}}\left(\left\{\mathbf{r}_{\uparrow}, \mathbf{r}_{\downarrow}\right\}\right)=\operatorname{det}\left(\tilde{g}_{i j}\right),
$$

where $i$ and $j$ run though the coordinates of all spin up and down electrons in $\left\{\mathbf{r}_{\uparrow}, \mathbf{r}_{\downarrow}\right\}$, respectively [3]. Note that the above equation is identical to Eq. (4) since $\tilde{g}_{i j}=\varphi\left(\mathbf{r}_{i}-\mathbf{r}_{j}\right)$.

Now, let us go back and address what happens if $u_{\mathbf{k}}=0$ at some momenta, which can be the case for the $d$-wave pairing symmetry. In this situation, Eq. (A3) is no longer valid. As shown below, special care must be taken for the momentum states with $u_{\mathbf{k}}=0$.

Specifically, imagine that $u_{\mathbf{k}}=0$ at $\mathbf{k}=\overline{\mathbf{k}}$. If so, the momentum states at $\overline{\mathbf{k}}$ and $-\overline{\mathbf{k}}$ are fully occupied by spin up and down electrons, respectively, which are completely decoupled from the rest of electrons forming Cooper pairs. For convenience, let us call these states the "preoccupied" momentum states.

Then, after some algebra, one can show that the amplitude of the RVB state is given as follows:

$$
\begin{aligned}
\psi_{\mathrm{RVB}}\left(\left\{\mathbf{r}_{\uparrow}, \mathbf{r}_{\downarrow}\right\}\right)= & \sum_{\forall \operatorname{comb} .}(-1)^{p} \operatorname{det}\left(\tilde{g}_{i j}\right) \\
& \times \operatorname{det}\left(e^{i \overline{\mathbf{k}}_{l} \cdot \overline{\mathbf{r}}_{m \uparrow}}\right) \operatorname{det}\left(e^{-i \overline{\mathbf{k}}_{l} \cdot \overline{\mathbf{r}}_{n \downarrow}}\right),
\end{aligned}
$$

where $\overline{\mathbf{k}}_{l}$ is the $l$ th preoccupied momentum, and $\overline{\mathbf{r}}_{m \uparrow}$ and $\overline{\mathbf{r}}_{n \downarrow}$ are the coordinates of the $m$-th spin-up and $n$th spin-down electrons, respectively, belonging to the preoccupied momentum states. Note that the number of preoccupied momenta is the same as that of spin up/down electrons belonging to the preoccupied momentum states. The sum is taken over all possible combinations of choosing $\left\{\overline{\mathbf{r}}_{\uparrow}, \overline{\mathbf{r}}_{\downarrow}\right\}$ out of $\left\{\mathbf{r}_{\uparrow}, \mathbf{r}_{\downarrow}\right\} . p$ is the permutation parity required for the rearrangement of all creation operators in a predetermined convention.

Unfortunately, however, computing Eq. (A4) turns out to be quite time-consuming due to the large number of different combinations. To reduce the computing time, we employ the trick of adding a very small constant to the pairing amplitude, i.e., $\Delta_{\mathbf{k}} \rightarrow \Delta_{\mathbf{k}}+\delta$, which nominally eliminates the preoccupied momentum states. As a matter of principle, this trick 
should generate the correct RVB state with the $d$-wave pairing symmetry in the limit of vanishing $\delta$. We have explicitly confirmed that the above trick works well for sufficiently small $\delta$. In Figs. 5 and 6 , we take $\delta / t=0.0001$.

\section{APPENDIX B: POINT GROUP SYMMETRY}

The group theoretical analysis can facilitate exact diagonalization of the Hamiltonian by categorizing the basis states into appropriate symmetry sectors block-diagonalizing the Hamiltonian.

Our specific goal is to understand the point group symmetries of the $t-J$ and projected BCS Hamiltonians in the square or triangular lattice. Here, we focus only on the point group symmetries since we are interested in the sub-Hilbert space with zero total momentum. Also, being a local operator, the Gutzwiller projection commutes with all point group operators. Thus we do not need to consider the effect of the Gutzwiller projection in our analysis of the point group symmetries.

Let us first discuss the point group symmetries in the square lattice. The basic point group in the square lattice is given by $\mathcal{C}_{4 \mathrm{v}}=\left\{e, C_{4}, C_{2}, C_{4}^{3}, \sigma_{x}, \sigma_{y}, \sigma_{\mathrm{d}}^{+}, \sigma_{\mathrm{d}}^{-}\right\}$, where $e$ is the identity operator, $C_{n}$ the rotation operator about the $z$ axis by the angle of $2 \pi / n, \sigma_{x / y}$ the reflection operator with respect to the $x / y$ axis, and $\sigma_{\mathrm{d}}^{+/-}$the reflection operator with respect to the $y=$ $\pm x$ line. With spin degrees of freedom, the point group should be enlarged to include the magnetic (or color) group. In other words, the spin-flip operator $f$ should be considered as a part of the point group.

Specifically, the point group of the $t-J$ model in the square lattice is determined by considering that both $H_{t}$ and $H_{J}$ commute with all the symmetry operators in $\mathcal{C}_{4 \mathrm{v}}$ as well as the spin-flip operator $f$. In other words, the $t-J$ model has $\mathcal{C}_{4 \mathrm{v}}^{\mathrm{II}} \equiv\{e, f\} \otimes \mathcal{C}_{4 \mathrm{v}}$ as its point group in the square lattice.

Meanwhile, the point group of the projected BCS Hamiltonian depends on the pairing symmetry. To understand this, it is important to note that $H_{\Delta}$ does not commute with some of the symmetry operators in $\mathcal{C}_{4 \mathrm{v}}^{\mathrm{II}}$, while both $H_{t}$ and $H_{\mu}$ do so with all of them. Specifically, $f H_{\Delta} f^{-1}=-H_{\Delta}$ for both $s$ and $d$-wave pairing symmetries, while $C_{4} H_{\Delta} C_{4}^{-1}=-H_{\Delta}$ for the $d$-wave pairing symmetry in the square lattice.

Consequently, in the square lattice, the point group of the projected BCS Hamiltonian with the $s$-wave pairing symmetry is simply given by $\mathcal{C}_{4 \mathrm{v}}^{\mathrm{I}} \equiv \mathcal{C}_{4 \mathrm{v}}$, excluding the spin-flip operator. For the $d$-wave pairing symmetry, however, the spin-flip operator can be combined with some of the symmetry operators in $\mathcal{C}_{4 \mathrm{v}}$. As a result, it can be shown that the point group of the projected BCS Hamiltonian with the $d$-wave pairing symmetry is given by $\mathcal{C}_{4 \mathrm{v}}^{\mathrm{III}} \equiv\left\{e, C_{2}, \sigma_{x}, \sigma_{y}, f C_{4}, f C_{4}^{3}, f \sigma_{\mathrm{d}}^{+}, f \sigma_{\mathrm{d}}^{-}\right\}$in the square lattice.

Now, it is important to note that the point group of the projected BCS Hamiltonian $\left(\mathcal{C}_{4 \mathrm{v}}^{\mathrm{I}}\right.$ and $\mathcal{C}_{4 \mathrm{v}}^{\mathrm{III}}$ for the $s$ - and $d$-wave pairing symmetries, respectively) is only the subgroup of that of the $t-J$ Hamiltonian $\left(\mathcal{C}_{4 \mathrm{v}}^{\mathrm{II}}\right)$. In general, if any two Hamiltonians have different point group symmetries, the overlap between their ground states would be greatly suppressed.

Fortunately, however, both effects of $f$ and $C_{4}$ on $H_{\Delta}$ amounts to simply changing the sign of the pairing amplitude $\Delta$. Note that such a sign change affects only the relative phase between the number-projected components of the BCS state belonging to different particle number sectors, while leaving the overall structure intact. This means that the overlap between the exact ground states of the $t-J$ model and the projected BCS theory can become substantial despite the nominal difference in their point group symmetries.

Finally, let us consider the point group symmetries in the triangular lattice. The basis point group in the triangular lattice is given by $\mathcal{C}_{6 \mathrm{v}}$. Since $f$ still works as a symmetry element for the $t-J$ model, the point group of the $t-J$ model is given by $\mathcal{C}_{6 \mathrm{v}}^{\mathrm{II}} \equiv\{e, f\} \otimes \mathcal{C}_{6 \mathrm{v}}$. Similar to the square lattice, the point group of the projected BCS Hamiltonian with the $s$-wave pairing symmetry is given by $\mathcal{C}_{6 \mathrm{v}}^{\mathrm{I}} \equiv \mathcal{C}_{6 \mathrm{v}}$ in the triangular lattice, excluding the spin-flip symmetry. Meanwhile, the projected BCS Hamiltonian with the $d$-wave pairing symmetry has only $\mathcal{C}_{2}$ as its point group in the triangular lattice.

\section{APPENDIX C: PROOF OF THE EQUIVALENCE AT HALF FILLING}

Any energy eigenstate of the projected BCS theory can be expanded in terms of its number-projected component in each particle number sector:

$$
|\psi\rangle=\sum_{h=0}^{N_{s}} \lambda_{h}\left|\phi_{h}\right\rangle,
$$

where $h$ is the number of holes, related with that of sites, $N_{s}$, and that of electrons, $N_{e}$, via $h=N_{s}-N_{e}$. $\lambda_{h}$ is the weighting amplitude in each particle number sector. Note that $h$ is always an even number since we are interested in the paired state.

We begin by writing the Schrödinger equation for the projected BCS theory:

$$
H_{\mathrm{PBCS}}|\psi\rangle=E|\psi\rangle,
$$

where the projected BCS Hamiltonian is decomposed as follows:

$$
H_{\mathrm{PBCS}}=\mathcal{H}_{t}+\mathcal{H}_{c}+\mathcal{H}_{a}-\mu N,
$$

where $\mathcal{H}_{t, c, a}=\mathcal{P}_{\mathrm{G}} H_{t, c, a} \mathcal{P}_{\mathrm{G}}$ with $H_{t}$ given in Eq. (10) and $H_{c}$ and $H_{a}$ being the creation and annihilation parts of $H_{\Delta}$ given in Eq. (10), respectively. The particle number operator is defined as $N=\sum_{i} n_{i}$.

Now, let us rewrite the Schrödinger equation component by component as follows: (i) for $h=0$,

$$
\lambda_{2} \mathcal{H}_{c}\left|\phi_{2}\right\rangle=\lambda_{0}\left(E+N_{s} \mu\right)\left|\phi_{0}\right\rangle ;
$$

(ii) for $2 \leqslant h \leqslant N_{s}-2$,

$$
\begin{aligned}
& \lambda_{h} \mathcal{H}_{t}\left|\phi_{h}\right\rangle+\lambda_{h+2} \mathcal{H}_{c}\left|\phi_{h+2}\right\rangle+\lambda_{h-2} \mathcal{H}_{a}\left|\phi_{h-2}\right\rangle \\
& \quad=\lambda_{h}\left(E+\mu\left(N_{s}-h\right)\right)\left|\phi_{h}\right\rangle ;
\end{aligned}
$$

and (iii) for $h=N_{s}$,

$$
\lambda_{N_{s}-2} \mathcal{H}_{a}\left|\phi_{N_{s}-2}\right\rangle=\lambda_{N_{s}} E\left|\phi_{N_{s}}\right\rangle .
$$

To begin with, it is important to note that half filling can be achieved by taking the limit of large chemical potential, $\mu \rightarrow \infty$. In this limit, Eq. (C4) requires the energy eigenvalue $E$ to scale as $E=-N_{s} \mu+\mathcal{O}\left(1 / \mu^{\alpha}\right)$ with $\alpha \geqslant 0$. This scaling behavior is obtained due to the fact that $\lambda_{0}$ and $\lambda_{2}$, for that 
matter, any $\lambda_{h}$ cannot diverge since they are the coefficients of the normalized wave function.

Meanwhile, combined with the above scaling behavior of $E$, Eq. (C6) dictates $\mathcal{O}\left(\lambda_{N_{s}-2}\right)=\mathcal{O}\left(E \lambda_{N_{s}}\right)=\mathcal{O}\left(\mu \lambda_{N_{s}}\right)$. Assuming that $\lambda_{h}$ decays as a power-law function of $\mu$, i.e., $\lambda_{h}=$ $\mathcal{O}\left(1 / \mu^{\nu_{h}}\right)$ in the limit of $\mu \rightarrow \infty$, the two decay exponents, $v_{N_{s}}$ and $v_{N_{s}-2}$, are related via $v_{N_{s}}=v_{N_{s}-2}+1$. Similarly, the simultaneous satisfaction of both Eqs. (C5) and (C6) requires $v_{h}=v_{h-2}+1$ for all $h \geqslant 2$.

This recursive relation for the decay exponents can be solved by noting that the zeroth decay exponent is actually zero, i.e., $v_{0}=0$. That is, $h_{0}=\mathcal{O}(1)$ in the limit of $\mu \rightarrow \infty$, maintaining the normalization of the wave function. Then, according to the recursive relation, $v_{h}=h / 2$. This means that $\lambda_{h}$ has the following scaling behavior:

$$
\lambda_{h}=\mathcal{O}\left(\frac{1}{\mu^{h / 2}}\right),
$$

which, in turn, sets $\alpha=1$ in the scaling behavior of $E$, when plugged into Eq. (C4). Consequently, $E$ has the following scaling behavior:

$$
E=-N_{s} \mu+\xi / \mu+\mathcal{O}\left(1 / \mu^{\beta}\right),
$$

where $\xi$ is a constant, which is yet to be determined, and $\beta$ is another decay exponent, which is larger than unity.

Now, by using the scaling behaviors of $\lambda_{h}$ and $E$, we can rewrite the component-by-component equations for $h=0$ and 2 by keeping only the most dominant terms:

$$
\begin{aligned}
\lambda_{2} \mathcal{H}_{c}\left|\phi_{2}\right\rangle & =\lambda_{0} \frac{\xi}{\mu}\left|\phi_{0}\right\rangle, \\
\lambda_{0} \mathcal{H}_{a}\left|\phi_{0}\right\rangle & =-2 \lambda_{2} \mu\left|\phi_{2}\right\rangle,
\end{aligned}
$$

which can be then combined to generate the Schrödinger equation solely at $h=0$ :

$$
\mathcal{H}_{c} \mathcal{H}_{a}\left|\phi_{0}\right\rangle=-2 \xi\left|\phi_{0}\right\rangle .
$$

Finally, under the no-double occupancy constraint, it can be shown that

$$
\begin{aligned}
\mathcal{H}_{c} \mathcal{H}_{a}\left|\phi_{0}\right\rangle & =\Delta^{2} \sum_{\langle i, j\rangle}\left(c_{i \uparrow}^{\dagger} c_{j \downarrow}^{\dagger}-c_{i \downarrow}^{\dagger} c_{j \uparrow}^{\dagger}\right)\left(c_{j \downarrow} c_{i \uparrow}-c_{j \uparrow} c_{i \downarrow}\right)\left|\phi_{0}\right\rangle \\
& =-2 \Delta^{2} \sum_{\langle i, j\rangle}\left(\mathbf{S}_{i} \cdot \mathbf{S}_{j}-n_{i} n_{j} / 4\right)\left|\phi_{0}\right\rangle, \quad(\mathrm{C} 12)
\end{aligned}
$$

where the second line is obtained by examining the matrix elements for all possible spin configurations in the $(i, j)$ sites, i.e., $|\uparrow \uparrow\rangle,|\uparrow \downarrow\rangle,|\downarrow \uparrow\rangle,|\downarrow \downarrow\rangle$. Consequently, the final Schrödinger equation at half filling becomes as follows:

$$
\Delta^{2} \sum_{\langle i, j\rangle}\left(\mathbf{S}_{i} \cdot \mathbf{S}_{j}-n_{i} n_{j} / 4\right)\left|\phi_{0}\right\rangle=\xi\left|\phi_{0}\right\rangle
$$

which is nothing but the Hamiltonian of the Heisenberg model.

Note that one of the authors have previously used a different analytical approach to prove the equivalence between the $t-J$ and projected BCS Hamiltonians at half filling. In this approach [14], the projected BCS Hamiltonian is replaced by the BCS Hamiltonian with finite on-site repulsive interaction $U$, dubbed as the $\mathrm{BCS}+U$ Hamiltonian. Eventually, the equivalence is proved by taking the limit of large $U$. Also, it is worthwhile to mention that, inspired by this approach, an alternative proof of the equivalence has been also put forward by using the path integral formalism [42].

\section{APPENDIX D: EXPLICIT ANALYSIS OF THE $2 \times 2$ SYSTEM}

Focusing on zero momentum (i.e., translationally invariant) and zero $z$ component of the total spin (i.e., spin-flip invariant), the entire Hilbert space of the $2 \times 2$ system can be expanded by the following seven basis states: (i) three states in the half-filled sector,

$$
\begin{aligned}
& \left|e_{1}\right\rangle=\frac{1}{\sqrt{2}}\left(\left|\begin{array}{ll}
\uparrow & \downarrow \\
\downarrow & \uparrow
\end{array}\right|+\left|\begin{array}{cc}
\downarrow & \uparrow \\
\uparrow & \downarrow
\end{array}\right|\right), \\
& \left|e_{2}\right\rangle=\frac{1}{2}\left(\left|\begin{array}{ll}
\uparrow & \uparrow \\
\downarrow & \downarrow
\end{array}\right|+\left|\begin{array}{cc}
\downarrow & \downarrow \\
\uparrow & \uparrow
\end{array}\right|+\left|\begin{array}{ll}
\downarrow & \uparrow \\
\downarrow & \uparrow
\end{array}\right|+\left|\begin{array}{ll}
\uparrow & \downarrow \\
\uparrow & \downarrow
\end{array}\right|\right), \\
& \left|e_{3}\right\rangle=\frac{1}{2}\left(\left|\begin{array}{ll}
\uparrow & \uparrow \\
\downarrow & \downarrow
\end{array}\right|+\left|\begin{array}{cc}
\downarrow & \downarrow \\
\uparrow & \uparrow
\end{array}\right|-\left|\begin{array}{ll}
\downarrow & \uparrow \\
\downarrow & \uparrow
\end{array}\right|-\left|\begin{array}{ll}
\uparrow & \downarrow \\
\uparrow & \downarrow
\end{array}\right|\right) ;
\end{aligned}
$$

(ii) three states in the two-hole sector,

$$
\begin{aligned}
& \left|e_{4}\right\rangle=\frac{1}{2}\left(\left|\begin{array}{ll}
0 & \uparrow \\
\downarrow & 0
\end{array}\right|+\left|\begin{array}{cc}
\uparrow & 0 \\
0 & \downarrow
\end{array}\right|-\left|\begin{array}{ll}
\downarrow & 0 \\
0 & \uparrow
\end{array}\right|-\left|\begin{array}{ll}
0 & \downarrow \\
\uparrow & 0
\end{array}\right|\right), \\
& \left|e_{5}\right\rangle=\frac{1}{\sqrt{8}}\left(\left|\begin{array}{ll}
0 & 0 \\
\downarrow & \uparrow
\end{array}\right|-\left|\begin{array}{ll}
0 & 0 \\
\uparrow & \downarrow
\end{array}\right|+\left|\begin{array}{cc}
\downarrow & \uparrow \\
0 & 0
\end{array}\right|-\left|\begin{array}{cc}
\uparrow & \downarrow \\
0 & 0
\end{array}\right|\right. \\
& \left.+\left|\begin{array}{ll}
\uparrow & 0 \\
\downarrow & 0
\end{array}\right|-\left|\begin{array}{ll}
\downarrow & 0 \\
\uparrow & 0
\end{array}\right|+\left|\begin{array}{ll}
0 & \uparrow \\
0 & \downarrow
\end{array}\right|-\left|\begin{array}{cc}
0 & \downarrow \\
0 & \uparrow
\end{array}\right|\right), \\
& \left|e_{6}\right\rangle=\frac{1}{\sqrt{8}}\left(\left|\begin{array}{ll}
0 & 0 \\
\downarrow & \uparrow
\end{array}\right|-\left|\begin{array}{ll}
0 & 0 \\
\uparrow & \downarrow
\end{array}\right|+\left|\begin{array}{cc}
\downarrow & \uparrow \\
0 & 0
\end{array}\right|-\left|\begin{array}{ll}
\uparrow & \downarrow \\
0 & 0
\end{array}\right|\right. \\
& \left.-\left|\begin{array}{ll}
\uparrow & 0 \\
\downarrow & 0
\end{array}\right|+\left|\begin{array}{ll}
\downarrow & 0 \\
\uparrow & 0
\end{array}\right|-\left|\begin{array}{ll}
0 & \uparrow \\
0 & \downarrow
\end{array}\right|+\left|\begin{array}{ll}
0 & \downarrow \\
0 & \uparrow
\end{array}\right|\right) ;
\end{aligned}
$$

and finally (iii) one state in the vacuum sector,

$$
\left|e_{7}\right\rangle=\left|\begin{array}{ll}
0 & 0 \\
0 & 0
\end{array}\right\rangle
$$

In terms of the ordered basis set $\left\{\left|e_{1}\right\rangle,\left|e_{2}\right\rangle,\left|e_{3}\right\rangle\right\}$, the $t-J$ Hamiltonian can be written in the half-filled sector as follows:

$$
H_{t-J}=2 J\left(\begin{array}{ccc}
-2 & \sqrt{2} & 0 \\
\sqrt{2} & -1 & 0 \\
0 & 0 & -1
\end{array}\right),
$$

generating the following ground state:

$$
\left|\phi_{0}^{t-J}\right\rangle=\mathcal{N}_{0}\left(\left|e_{1}\right\rangle-\frac{1}{\sqrt{2}}\left|e_{2}\right\rangle\right),
$$

where $\mathcal{N}_{0}=\sqrt{2 / 3}$ is the normalization constant. Note that $\left|e_{3}\right\rangle$ couples with neither $\left|e_{1}\right\rangle$ nor $\left|e_{2}\right\rangle$ since they have different point group symmetries. 
Similarly, in terms of the ordered basis set $\left\{\left|e_{4}\right\rangle,\left|e_{5}\right\rangle,\left|e_{6}\right\rangle\right\}$, the $t-J$ Hamiltonian can be written in the two-hole sector as follows:

$$
H_{t-J}=-2\left(\begin{array}{ccc}
0 & 2 \sqrt{2} t & 0 \\
2 \sqrt{2} t & J & 0 \\
0 & 0 & J
\end{array}\right),
$$

generating the following ground state:

$$
\left|\phi_{2}^{t-J}\right\rangle=\mathcal{N}_{2}\left(\alpha\left|e_{4}\right\rangle+\beta\left|e_{5}\right\rangle\right),
$$

where $\quad \alpha=\sqrt{J^{2}+32 t^{2}}-J, \quad \beta=4 \sqrt{2} t, \quad$ and $\quad \mathcal{N}_{2}=$ $1 / \sqrt{|\alpha|^{2}+|\beta|^{2}}$. Similar to half filling, $\left|e_{6}\right\rangle$ couples with neither $\left|e_{4}\right\rangle$ nor $\left|e_{5}\right\rangle$.

Meanwhile, the projected BCS Hamiltonian with the $d$-wave pairing symmetry can be written as the following block diagonal form in terms of the ordered basis set $\left\{\left|e_{1}\right\rangle,\left|e_{2}\right\rangle,\left|e_{4}\right\rangle,\left|e_{5}\right\rangle,\left|e_{3}\right\rangle,\left|e_{6}\right\rangle,\left|e_{7}\right\rangle\right\}$ :

$$
\begin{aligned}
H_{\mathrm{PBCS}}= & \left(\begin{array}{cccc}
-4 \mu & 0 & 0 & 4 \Delta \\
0 & -4 \mu & 0 & -2 \sqrt{2} \Delta \\
0 & -0 & -2 \mu & -4 \sqrt{2} t \\
4 \Delta & -2 \sqrt{2} \Delta & -4 \sqrt{2} t & -2 \mu
\end{array}\right) \\
& \oplus\left(\begin{array}{ccc}
-4 \mu & 2 \sqrt{2} \Delta & 0 \\
2 \sqrt{2} \Delta & -2 \mu & -4 \sqrt{2} \Delta \\
0 & -4 \sqrt{2} \Delta & 0
\end{array}\right) .
\end{aligned}
$$

The characteristic polynomial of each block matrix is given as follows:

$$
\begin{aligned}
\mathcal{C}_{-}(\varepsilon)= & (\varepsilon+4 \mu)\left[(\varepsilon+4 \mu)^{3}-4 \mu(\varepsilon+4 \mu)^{2}\right. \\
& \left.+4\left(\mu^{2}-6 \Delta^{2}-8 t^{2}\right)(\varepsilon+4 \mu)+48 \Delta^{2} \mu\right], \\
\mathcal{C}_{+}(\varepsilon)= & (\varepsilon+4 \mu)^{3}-6 \mu(\varepsilon+4 \mu)^{2} \\
& +8\left(\mu^{2}-5 \Delta^{2}\right)(\varepsilon+4 \mu)+32 \Delta^{2} \mu,
\end{aligned}
$$

where the subscript $-/+$ indicates the parity associated with the $\pi / 2$ rotation followed by the spin flip.

After a careful examination, one can show that the ground state occurs in the negative parity sector if $\mu$ gets sufficiently large. In this situation, it is convenient to observe that there is an easy solution of $\mathcal{C}_{-}(\varepsilon)$, whose eigenvalue is $-4 \mu$ with the corresponding eigenstate given by $\left|e_{1}\right\rangle+\sqrt{2}\left|e_{2}\right\rangle$. The other three eigenstates in the negative parity sector, including the ground state, should be orthogonal to this eigenstate. As a result, the ground state at sufficiently large $\mu$ takes the following form:

$$
\left|\psi_{\mathrm{PBCS}}\right\rangle=\mathcal{N}\left(\left|e_{1}\right\rangle-\frac{1}{\sqrt{2}}\left|e_{2}\right\rangle+f\left|e_{4}\right\rangle+g\left|e_{5}\right\rangle\right),
$$

where $\mathcal{N}=1 / \sqrt{3 / 2+|f|^{2}+|g|^{2}}$ is the normalization constant, and $f$ and $g$ are some appropriate functions of $\Delta / t$ and $\mu / t$, whose details are not provided here except that $|f|,|g| \ll 1$ for sufficiently large $\mu$. It is important to note that the half-filled component of $\left|\psi_{\mathrm{PBCS}}\right\rangle,\left|e_{1}\right\rangle-\frac{1}{\sqrt{2}}\left|e_{2}\right\rangle$, coincides exactly with the ground state of $t-J$ model in the same sector,
$\left|\phi_{0}^{t-J}\right\rangle$, as expected from the proof of the equivalence at half filling.

Actually, it is interesting to perform the similar analysis for the projected BCS theory with the $s$-wave pairing symmetry, whose ground state also coincides exactly with the ground state of $t-J$ model at half filling for basically the same reason mentioned above. Thus there is a certain degree of robustness in the equivalence between the $t-J$ model and the projected BCS theory at half filling.

Now, an important question is if $\left|\psi_{\mathrm{PBCS}}\right\rangle$ can still accurately capture the ground state of $t-J$ model as $\mu$ is reduced. In other words, how accurately $f$ and $g$ in Eq. (D10) can capture $\alpha$ and $\beta$ in Eq. (D7) as a function of $\mu$ ? This question can be answered by computing the NWOS:

$$
\overline{\mathcal{O}^{2}}=\mathcal{N}^{2}\left(\frac{3}{2}+\frac{\left|f^{*} \alpha+g^{*} \beta\right|^{2}}{|\alpha|^{2}+|\beta|^{2}}\right) .
$$

As one can see from Fig. 1(a) at $N_{s}=4$, the NWOS remains high upon doping. Note that there is a phase transition along the certain critical line of $\mu$ and $\Delta$, beyond which the ground state of the projected BCS theory is no longer given by Eq. (D10).

While providing various successful small-system checks, the $2 \times 2$ system is way too small to judge if the optimal pairing amplitude can form a well-defined nonzero curve as a function of doping. To this end, one has to analyze larger systems via full-fledged exact diagonalization.

\section{APPENDIX E: TESSELLATION OF THE LATTICE}

We begin with the tessellation of the square lattice, which can be tiled with tilted square unit cells. It is important to note that we focus only on the tilted square unit cells since they preserve the rotational symmetry of the square lattice.

Without loss of generality, the distance between any two adjacent tilted square unit cells can be written as $d_{\mathrm{sq}}=$ $\sqrt{n^{2}+m^{2}}$ (in units of lattice constant) for any non-negative integers $n$ and $m$, which is also the side length of the titled square unit cell. This means that the number of sites enclosed by the tilted square unit cell is $N_{s}=d_{\mathrm{sq}}^{2}=n^{2}+m^{2}$. Since we are interested in the systems with even number of particles with zero $z$ component of the total spin, both $m$ and $n$ should be either even or odd integers, which means $N_{s}=2,4,8,10,16,18,20,26,32,34,36, \ldots$.

For illustration, some of the tilted square unit cells are depicted in Fig. 10. Note that, if either $n$ or $m$ is zero (e.g., $N_{s}=4$ and 16), or $n=m$ (e.g., $N_{s}=8$ and 18), the tessellation with tilted square unit cells has additional reflection symmetries; one for the horizontal axis, one for the vertical axis, and two for the diagonal axes. That is, the point groups become $\mathcal{C}_{4 \mathrm{v}}$ for $N_{s}=4,8,16$, and 18 .

Due to the exponential growth of the Hilbert space, we are able to perform exact diagonalization only up to $N_{s}=20$ in this work. Figure 11 shows the number of basis states in the common $\operatorname{logarithm}$ scale, $\log N_{b}$, as a function of hole concentration $x=1-N_{e} / N_{s}$. Note that, here, the number of basis states is computed within the restricted Hilbert space, where both total momentum and $z$-component of the total spin are zero without using any other point group symmetries. As 


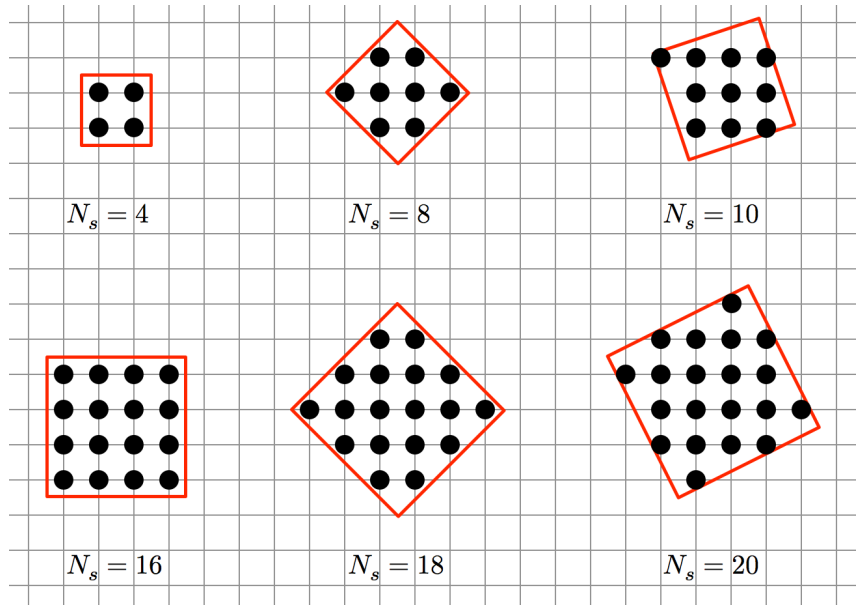

FIG. 10. Tessellation of the square lattice via tilted square unit cells. The point groups for $N_{s}=4,8,16,18$ and 10,20 are $\mathcal{C}_{4 \mathrm{v}}$ and $\mathcal{C}_{4}$, respectively.

one can see, the total number of basis states is more than 10 millions for $N_{s}=20$. Roughly speaking, the total number of states increases by one order of magnitude as the number of sites increases by two. This means that the next available system at $N_{s}=26$ would have more than 10 billion basis states, which are beyond the current computing capacity.

Now, let us switch gears to the tessellation of the triangular lattice. Similar to the square lattice, without loss of generality, the distance between any two adjacent tilted hexagonal unit cells can be written as $d_{\mathrm{tr}}=\sqrt{n^{2}+n m+m^{2}}$ (in units of lattice constant) for any non-negative integers $n$ and $m$. After a moment of deliberation, one can show that the number of sites enclosed by the tilted hexagonal unit cell is also given by $N_{s}=d_{\mathrm{tr}}^{2}=n^{2}+n m+m^{2}$. Both $m$ and $n$ should be even integers for $N_{s}$ to be an integer number, which means $N_{s}=4,12,16,28,36, \ldots$ Meanwhile, the $120^{\circ}$ spin order can exist only when $m=n(\bmod 3)$. Combined all together,

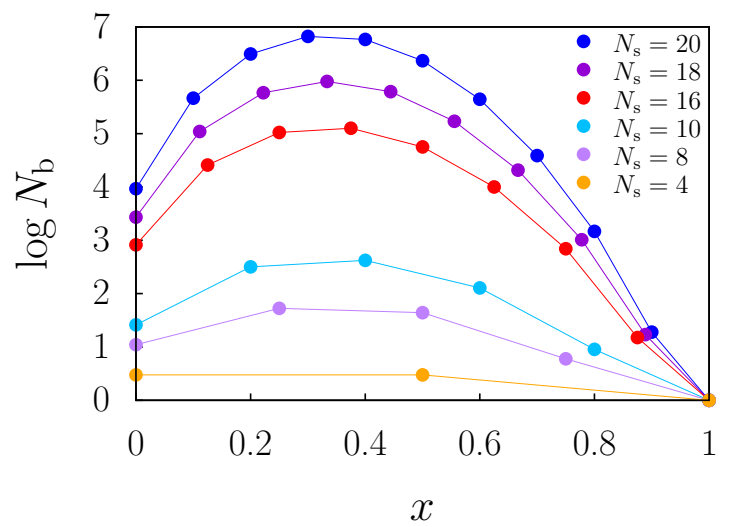

FIG. 11. Number of basis states for various tilted square unit cells as a function of hole concentration. Note that the ordinate is the number of basis states in the common logarithm scale, $\log N_{b}$. Here, the hole concentration is defined only discretely as $x=1-N_{e} / N_{s}$ with $N_{e}$ and $N_{s}$ being the numbers of electrons and sites, respectively. this means that the only accessible system in this work via exact diagonalization is that with $N_{s}=12$.

\section{APPENDIX F: VARIATION OF $J / t$}

Figure 12 shows how the NWOS between the exact ground states of the $t-J$ model and the projected BCS theory with the $d$-wave pairing symmetry evolves with the variation of $J / t$ in the square lattice. As one can see from Fig. 12(a), the NWOS is maximized along the line of $\Delta=0$ for small $J / t$, meaning that there is no pairing at this parameter regime.

The optimal pairing amplitude producing the maximum NWOS gets lifted from zero if $J / t$ becomes larger than a critical value, say 0.3 . As $J / t$ increases further, the optimal pairing amplitude also gets higher, expanding the regime of superconductivity.

Too large values of $J / t$ may not be physically meaningful in the single-band model, where the $t-J$ model is obtained as the large- $U$ expansion of the Hubbard model with $J / t$ being proportional to $t / U[31,32]$. It is, however, worthwhile

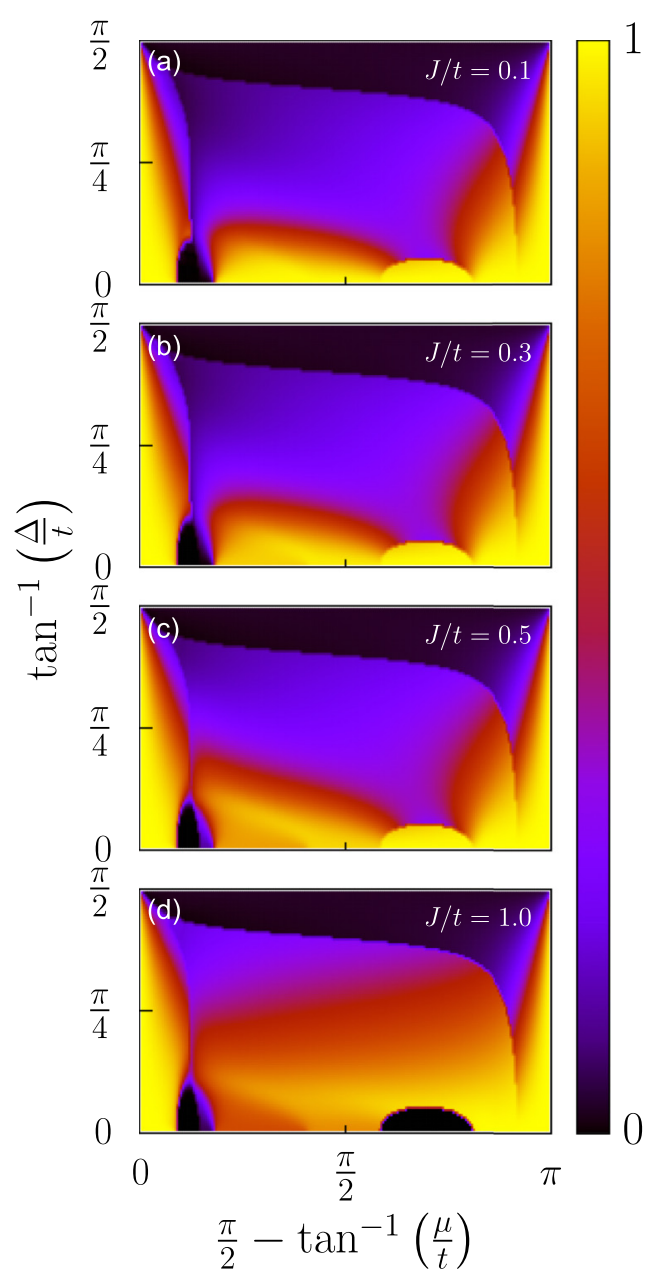

FIG. 12. Maps of the NWOS between the exact ground states of the $t-J$ model and the projected BCS theory with the $d$-wave pairing symmetry for various values of $J / t$ in the square lattice. The spin exchange coupling constant is varied as $J / t=0.1,0.3,0.5$, and 1.0 from (a) to (d). This result is obtained at $N_{s}=16$. 
to note that the spin exchange coupling can be also generated in the three-band model via the $d-p$ hybridization mech- anism, where $J / t$ does not follow the simple $t / U$ scaling [33].
[1] P. W. Anderson, The resonating valence bond state in $\mathrm{La}_{2} \mathrm{CuO}_{4}$ and superconductivity, Science 235, 1196 (1987).

[2] G. Baskaran, Z. Zou, and P. W. Anderson, The resonating valence bond state and high- $T_{c}$ superconductivity - A mean field theory, Solid State Commun. 63, 973 (1987).

[3] C. Gros, Superconductivity in correlated wave functions, Phys. Rev. B 38, 931 (1988).

[4] H. Yokoyama and H. Shiba, Variational Monte-Carlo studies of superconductivity in strongly correlated electron systems, J. Phys. Soc. Jpn. 57, 2482 (1988).

[5] A. Paramekanti, M. Randeria, and N. Trivedi, Projected Wave Functions and High Temperature Superconductivity, Phys. Rev. Lett. 87, 217002 (2001).

[6] S. Sorella, G. B. Martins, F. Becca, C. Gazza, L. Capriotti, A. Parola, and E. Dagotto, Superconductivity in the TwoDimensional $t$ - $J$ Model, Phys. Rev. Lett. 88, 117002 (2002).

[7] A. Paramekanti, M. Randeria, and N. Trivedi, High- $T_{c}$ superconductors: A variational theory of the superconducting state, Phys. Rev. B 70, 054504 (2004).

[8] P. W. Anderson, P. A. Lee, M. Randeria, T. M. Rice, N. Trivedi, and F. C. Zhang, The physics behind high-temperature superconducting cuprates: The 'Plain Vanilla' version of RVB, J. Phys.: Condens. Matter 16, R755 (2004).

[9] B. A. Bernevig, R. B. Laughlin, and D. I. Santiago, Magnetic Instability in Strongly Correlated Superconductors, Phys. Rev. Lett. 91, 147003 (2003).

[10] F. C. Zhang, Gossamer Superconductor, Mott Insulator, and Resonating Valence Bond State in Correlated Electron Systems, Phys. Rev. Lett. 90, 207002 (2003).

[11] T. Misawa and M. Imada, Origin of high- $T_{c}$ superconductivity in doped Hubbard models and their extensions: Roles of uniform charge fluctuations, Phys. Rev. B 90, 115137 (2014).

[12] S. Sachdev and K. Park, Ground states of quantum antiferromagnets in two dimensions, Ann. Phys. (NY) 298, 58 (2002).

[13] K. Park, Theoretical Evidence for Equivalence between the Ground States of the Strong Coupling BCS Hamiltonian and the Antiferromagnetic Heisenberg Model, Phys. Rev. Lett. 95, 027001 (2005).

[14] K. Park, Quantum antiferromagnetism and high $T_{c}$ superconductivity: A close connection between the $t-J$ model and the projected BCS Hamiltonian, Phys. Rev. B 72, 245116 (2005).

[15] E. Demler, W. Hanke, and S. C. Zhang, $S O(5)$ theory of antiferromagnetism and superconductivity, Rev. Mod. Phys. 76, 909 (2004).

[16] J. K. Jain, Composite-Fermion Approach for the Fractional Quantum Hall Effect, Phys. Rev. Lett. 63, 199 (1989).

[17] J. K. Jain, Composite Fermions (Cambridge University Press, Cambridge, England, 2007).

[18] G. Moore and N. Read, Nonabelions in the fractional quantum hall effect, Nucl. Phys. B 360, 362 (1991).

[19] M. Greiter, X. G. Wen, and F. Wilczek, Paired hall states, Nucl. Phys. B 374, 567 (1992).
[20] K. Park, V. Melik-Alaverdian, N. E. Bonesteel, and J. K. Jain, Possibility of p-Wave pairing of composite fermions at $v=1 / 2$, Phys. Rev. B 58, R10167 (1998).

[21] V. W. Scarola, K. Park, and J. K. Jain, Cooper instability of composite fermions, Nature (London) 406, 863 (2000).

[22] H. Lu, S. Das Sarma, and K. Park, Superconducting order parameter for the even-denominator fractional quantum hall effect, Phys. Rev. B 82, 201303(R) (2010).

[23] R. Willett, J. P. Eisenstein, H. L. Störmer, D. C. Tsui, A. C. Gossard, and J. H. English, Observation of an EvenDenominator Quantum Number in the Fractional Quantum Hall Effect, Phys. Rev. Lett. 59, 1776 (1987).

[24] For review, see P. A. Lee, N. Nagaosa, and X.-G. Wen, Doping a Mott insulator: Physics of high-temperature superconductivity, Rev. Mod. Phys. 78, 17 (2006).

[25] A. Lopez and E. Fradkin, Fractional quantum hall effect and chern-simons gauge theories, Phys. Rev. B 44, 5246 (1991).

[26] V. Kalmeyer and S. C. Zhang, Metallic phase of the quantum hall system at even-denominator filling fractions, Phys. Rev. B 46, 9889(R) (1992).

[27] B. I. Halperin, P. A. Lee, and N. Read, Theory of the half-filled landau level, Phys. Rev. B 47, 7312 (1993).

[28] E. H. Rezayi and F. D. M. Haldane, Incompressible Paired Hall State, Stripe Order, and the Composite Fermion Liquid Phase in Half-Filled Landau Levels, Phys. Rev. Lett. 84, 4685 (2000).

[29] M. R. Peterson, K. Park, and S. Das Sarma, Spontaneous Particle-Hole Symmetry Breaking in the $v=5 / 2$ Fractional Quantum Hall Effect, Phys. Rev. Lett. 101, 156803 (2008).

[30] J.-S. Jeong and K. Park, Bilayer mapping of the paired quantum Hall state: Instability toward anisotropic pairing, Phys. Rev. B 91, 195119 (2015)

[31] C. Gros, R. Joynt, and T. M. Rice, Superconducting Instability in the Large- $U$ Limit of the Two-Dimensional Hubbard Model, Z. Phys. B: Condens. Matter 68, 425 (1987).

[32] A. H. MacDonald, S. M. Girvin, and D. Yoshioka, $t / U$ expansion for the Hubbard model, Phys. Rev. B 37, 9753 (1988).

[33] F. C. Zhang and T. M. Rice, Effective Hamiltonian for the superconducting Cu oxides, Phys. Rev. B 37, 3759(R) (1988).

[34] J. Oitmaa and D. D. Betts, The ground state of two quantum models of magnetism, Can. J. Phys. 56, 897 (1978).

[35] J. D. Reger and A. P. Young, Monte Carlo simulations of the spin-1/2 Heisenberg antiferromagnet on a square lattice, Phys. Rev. B 37, 5978(R) (1988).

[36] S. Tang and J. E. Hirsch, Long-range order without broken symmetry: Two-dimensional Heisenberg antiferromagnet at zero temperature, Phys. Rev. B 39, 4548 (1989).

[37] T. Barnes, The 2D Heisenberg antiferromagnet in high-Tc superconductivity: A review of numerical techniques and results, Int. J. Mod. Phys. C 2, 659 (1991).

[38] E. Manousakis, The spin-1/2 Heisenberg antiferromagnet on a square lattice and its application to the cuprous oxides, Rev. Mod. Phys. 63, 1 (1991). 
[39] E. Dagotto, Correlated electrons in high-temperature superconductors, Rev. Mod. Phys. 66, 763 (1994).

[40] Y.-L. Wu, N. Regnault, and B. A. Bernevig, Gauge-fixed Wannier wave functions for fractional topological insulators, Phys. Rev. B 86, 085129 (2012).
[41] P. W. Anderson, Resonating valence bonds: A new kind of insulator?, Mater. Res. Bull. 8, 153 (1973).

[42] E. Kochetov, A. Ferraz, and R. T. Pepino, Low-energy representation of the projected BCS Hamiltonian close to half filling, Phys. Rev. B 79, 115135 (2009). 